\title{
Receptivity of Hypersonic Boundary Layers to Acoustic and Vortical Disturbances (Invited)
}

\author{
P. Balakumar ${ }^{1}$ \\ Flow Physics and Control Branch \\ NASA Langley Research Center, Hampton, VA 23581
}

\begin{abstract}
Boundary-layer receptivity to two-dimensional acoustic and vortical disturbances for hypersonic flows over two-dimensional and axi-symmetric geometries were numerically investigated. The role of bluntness, wall cooling, and pressure gradients on the receptivity and stability were analyzed and compared with the sharp nose cases. It was found that for flows over sharp nose geometries in adiabatic wall conditions the instability waves are generated in the leading-edge region and that the boundary layer is much more receptive to slow acoustic waves as compared to the fast waves. The computations confirmed the stabilizing effect of nose bluntness and the role of the entropy layer in the delay of boundarylayer transition. The receptivity coefficients in flows over blunt bodies are orders of magnitude smaller than that for the sharp cone cases. Wall cooling stabilizes the first mode strongly and destabilizes the second mode. However, the receptivity coefficients are also much smaller compared to the adiabatic case. The adverse pressure gradients increased the unstable second mode regions.
\end{abstract}

\section{Nomenclature}

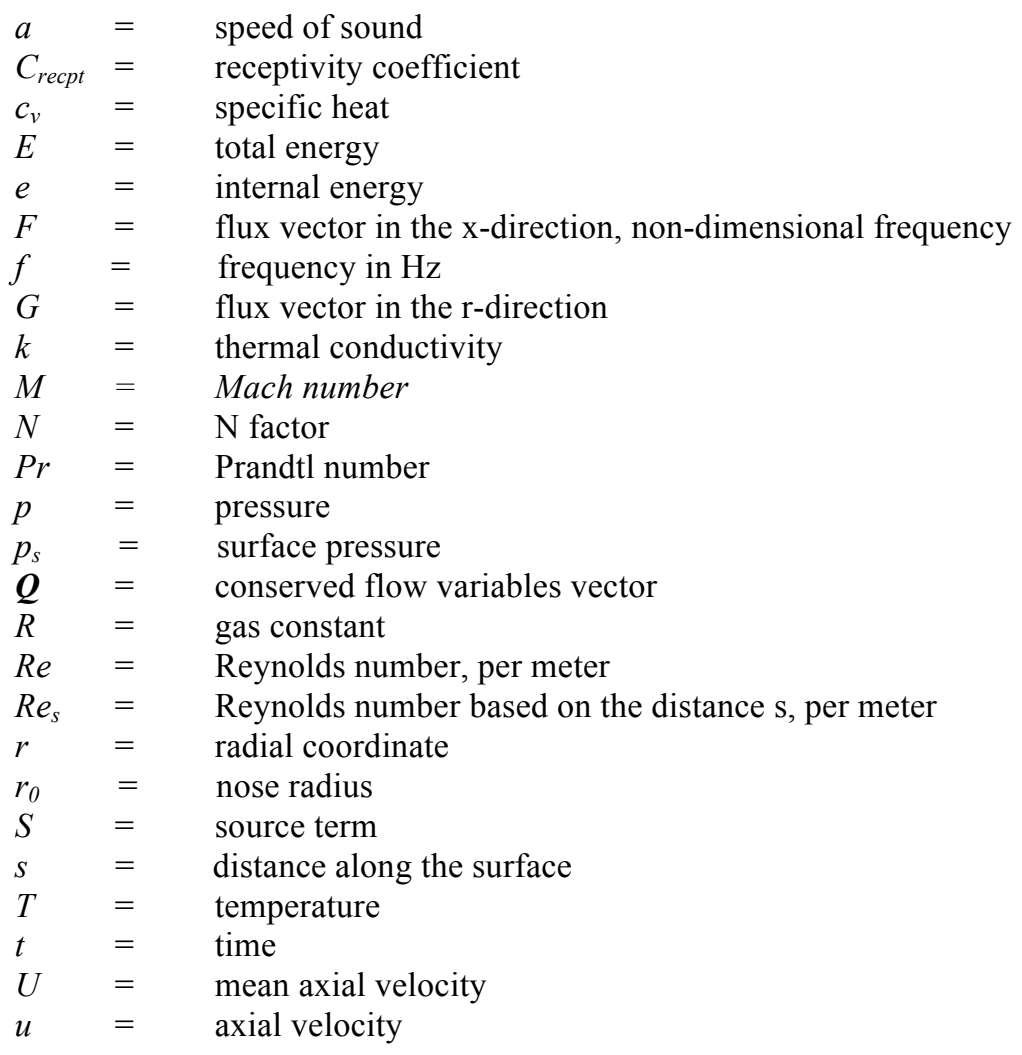

\footnotetext{
${ }^{1}$ Research Scientist, AIAA Associate Fellow
} 


$\begin{array}{lll}V & = & \text { radial velocity } \\ X & = & \text { axial coordinate } \\ x_{0} & = & \text { reference } \mathrm{x} \text { location } \\ \alpha & = & \text { wavenumber in the } \mathrm{x} \text {-direction } \\ \eta & = & \text { curvilinear coordinate in the radial direction, boundary-layer similarity coordinate } \\ \delta & = & \text { boundary layer thickness } \\ \mu & = & \text { viscosity } \\ \xi & = & \text { curvilinear coordinate in the axial direction } \\ \rho & = & \text { density } \\ \omega & = & \text { frequency in radian }\end{array}$

$\begin{array}{lll}\text { Subscripts } & \\ a c \quad= & \text { acoustic } \\ e & = & \text { boundary-layer edge conditions } \\ \max = & \text { maximum } \\ n & = & \text { normal to the surface, neutral point } \\ \infty & = & \text { freestream quantities }\end{array}$

\title{
I. Introduction
}

Accurate prediction of transition onset and transition end points, as well as modeling of the transitional and turbulent regions, are major concerns when using CFD codes to compute aerodynamic quantities such as lift, drag, skin friction, and heat transfer. Transition from a laminar to a turbulent state in shear flows occurs due to the evolution and interaction of different disturbances inside the shear layer. Though there are several mechanisms and routes to go from a laminar to a turbulent state, most of them in quiet environments generally follow these fundamental processes ${ }^{1}$ :

\author{
- Receptivity \\ - Linear instability \\ - $\quad$ Nonlinear instability and saturation \\ - $\quad$ Secondary instability and breakdown to turbulence
}

In the receptivity process, unsteady disturbances in the environment such as acoustic, entropy, and turbulence interact with the boundary layer and/or with the inhomogeneities in the geometry such as roughness and generate instability waves inside the shear layer. In quiet environments, the initial amplitudes of these instability waves are small compared to any characteristic velocity, density, and temperature scales in the flow. In the second stage, the amplitudes of these instability waves grow exponentially downstream and this process is governed by the linearized Navier-Stokes equation. Further downstream, the amplitudes of the disturbances become large and the nonlinear effects inhibit the exponential growth and the amplitudes of the waves eventually saturate. In the next stage, these finite amplitude saturated disturbances become unstable to two- and/or three-dimensional disturbances. This is called secondary instability and beyond this stage the spectrum broadens, due to complex interactions and further instabilities, and the flow becomes turbulent in a short distance downstream. When the disturbances reach large amplitudes due to the exponential growth, the nonlinear effects set in and the mean flow becomes distorted. Associated with this distortion, local skin friction and heat transfer rates increase from their respective laminar values. The location where they start to deviate from the laminar values is defined as the transition onset point. Hence the transition onset is determined by three factors: (1) the initial amplitudes of the instability waves, (2) the growth rates of the instability waves, and (3) the threshold value for the amplitude of the instability waves at which transition onset will trigger. The focus of this summary paper is in answering the first two factors in hypersonic flows over two-dimensional and axi-symmetric geometries. Specifically, we concentrate on what are the initial amplitudes of the instability waves generated by the freestream acoustic and vortical disturbances in hypersonic boundary layers over sharp and blunt wedges and straight and flared cones under adiabatic and cold wall conditions.

The stability characteristics of compressible two-dimensional and axi-symmetric boundary layers have been thoroughly investigated $^{2-5}$ numerically. Several experiments were performed to understand and validate the stability characteristics of hypersonic boundary layers over flat plates and cones. ${ }^{6-15}$ Stability characteristics of a boundary layer depend on several parameters including: boundary-layer edge Mach number $\left(M_{e}\right)$, local Reynolds number, 
pressure gradient, and wall conditions. The laminar boundary-layer profiles and its stability characteristics can be computed without much difficulty. The instability is governed by the linearized Navier-Stokes equations. The normal mode analysis of this equation yields an eigenvalue system that determines the stability characteristics of the boundary layer. The solution of this system yields several discrete and continuous eigensolutions. The eigensolutions corresponding to the continuous spectrum give the local forced response of the boundary layer to incoming or outgoing acoustic, vortical, or entropy disturbances. Without any local forcing, the contribution of the continuous spectrum to the boundary-layer disturbances does not exist. Among the discrete eigensolutions, only one or a few, at most, may be unstable. These unstable modes grow exponentially downstream and cause the boundary layer to transition to turbulence.

The main conclusions that are drawn from these investigations can be summarized as follows. (1) The quantity $\left(\rho U^{\prime}\right)^{\prime}=\frac{d}{d y}\left(\rho \frac{d U}{d y}\right)$, where $\rho$ and $U$ are the density and the streamwise velocity distributions of the mean flow and $y$ the coordinate normal to the wall, plays the same role in the compressible stability theory as $U^{\prime \prime}$ does in the incompressible theory. The location where $\left(\rho U^{\prime}\right)^{\prime}=0$ is called the "Generalized inflection point (G.I.P)." In most of the compressible boundary layers, the density profile has an inflection point $\rho^{\prime \prime}=0$ and due to this, compressible boundary layers exhibit generalized inflection points. As a consequence, the flat-plate/cone compressible boundary layers are unstable to purely inviscid disturbances. This is one of the differences between the instability of incompressible and compressible flows. (2) When the mean flow relative to the neutral disturbance phase velocity becomes supersonic over some portion of the boundary layer, there exist several unstable modes. For twodimensional disturbances, it is the first of the additional modes, called the second mode or the Mack mode, which is the most unstable at all Mach numbers for which the relative flow is supersonic. In the inviscid limit, the phase velocity of the instability disturbances varies from $1-1 / M_{e}$ to $C_{s}$, where $C_{s}$ is the mean flow velocity at which the generalized inflection $\left(\rho U^{\prime}\right)^{\prime}=0$ occurs.

The growth rates of the instability waves strongly depend on the wall conditions, nose bluntness, and pressure gradients along the surface. The effects of wall cooling on transition have been studied experimentally and numerically by many researchers. Wall cooling makes the boundary layer thinner compared to the adiabatic case. Lees ${ }^{16}$ predicted that cooling the surface would stabilize the boundary layer. Later, Mack's ${ }^{4}$ computational results showed that the first mode was stabilized by cooling; however, the higher modes were destabilized by this process. Experiments of Demetriades ${ }^{17}$ and Lysenko ${ }^{18}$ confirmed that cooling the wall increased the growth rates of the second mode disturbances and reduced the transition Reynolds number. Stetson ${ }^{19}$ investigated the effects of surface temperature on the stability of the laminar boundary layer experimentally and the results also verified linear stability theory. Blanchard ${ }^{9}$ investigated effects of cooling in the hypersonic boundary-layer transition over a flared cone in the NASA Langley Mach 6 Quiet Tunnel and showed that the most amplified frequency is higher in the cold wall case compared to the adiabatic case and also found that the transition moved upstream in the cold wall case.

The effects of nose tip bluntness on the stability and transition of hypersonic boundary layers have been investigated experimentally and numerically by many researchers ${ }^{20-37}$. The experiments showed that nose bluntness had a stabilizing effect upon the boundary layer and transition occurred further downstream compared to that for the sharp nose cases. Malik et al. ${ }^{22}$ computed the effect of nose bluntness on boundary-layer instability for Mach 8 flow past a 7-degree half-angle cone. They included the entropy-layer effect using Parabolized Navier-Stokes equations. They concluded that nose bluntness stabilizes the boundary layer and the effect of unit Reynolds number in the aeroballistic range data of Potter ${ }^{33}$ was a nose bluntness effect. Rosenboom et al. ${ }^{34}$ and Zhong ${ }^{35,36}$ did further studies on the effect of nose bluntness on the linear stability of hypersonic flow over Stetson's blunt cone and focused on the transition reversal phenomenon. However, no instability reversal was observed as the nose radius increased in both studies. Their results indicated that to understand the cause of the transition reversal phenomenon it is necessary to conduct further studies on the nose bluntness. Balakumar ${ }^{37}$ performed computations for a blunt flat plate with thicknesses from 0.0001 to 0.01 inches and a wedge of 10-degree half-angle with different leading-edge radii 0.001 and 0.01 inches to find out the effect of nose bluntness on the stability of two-dimensional boundary layers. He found that the bluntness has a strong stabilizing effect on the stability of two-dimensional boundary layers and the boundary layers on wedges are far more stable that on blunt flat plates.

Balakumar and Malik ${ }^{38}$ computed the parametric effects of the pressure gradient and wall cooling on the stability of the flow over flared-cones. A series of experiments ${ }^{9,10,13,14}$ were performed on flared-cones to investigate the effects of adverse pressure gradients on the stability of hypersonic boundary layers. The important conclusions are that adverse pressure gradients inhibit the growth of the boundary layer and hence the growth of the second modes remains almost constant for a long distance. This causes the flow to become transitional earlier as compared to the zero pressure gradient case. 
In order for an unsteady external forcing to excite an instability wave, both the frequency and the wavelength of the forcing disturbance must match with those of boundary-layer instability waves. The spatial scales of free-stream disturbances and boundary-layer instabilities are, in general, significantly disparate. Therefore, a wavelength conversion mechanism is needed for an external disturbance to excite an instability wave in a boundary layer. A strongly nonparallel mean flow, such as the leading-edge flow or that in the vicinity of a localized inhomogeneity, has all the required spatial scales for this conversion to take place. It was this realization that formed the basis of Goldstein' ${ }^{39,40}$ seminal work on generation of Tollmien-Schlichting waves by long wavelength free-stream disturbances in low-speed flows.

In hypersonic flows, the receptivity process becomes involved due to the existence of (1) a bow shock at the nose region and (2) multiple discrete modes and continuous spectrum in the stability characteristics of the boundary layer. The freestream disturbances consist of slow and fast acoustic waves, vorticity waves, entropy waves, and thermal spots. The analytical form of these waves can be obtained by solving the homogeneous linearized Euler equations. When one of these waves impinges on the shock, multiple waves may be generated across the shock downstream in addition to the transmission of the incident wave. ${ }^{41}$ Hence the boundary layer on the surface is forced by these multiple transmitted waves. The disturbances generated inside the boundary layer will depend on the frequency and wavelength of the transmitted waves and on the response of the boundary layer to these forcings.

There have been a number of investigations conducted on the interaction of acoustic waves with supersonic boundary layers. The interaction of acoustic waves with a supersonic boundary layer using inhomogeneous stability equations was investigated in Ref. [42-43]. One important finding was that due to the interaction, the acoustic waves excite disturbances inside the boundary layer, which are much larger than that in the free stream. The interaction of stream acoustic waves with a non-parallel boundary layer was studied in [44]. The analysis and the calculations showed that the disturbances inside the boundary layer reach values significantly higher compared to that in the free stream. It was also observed that there exists a critical Reynolds number where this excitation is the highest.

In hypersonic boundary layers, among all the discrete modes, the slow and the fast modes are the two relevant modes for boundary-layer transition. ${ }^{45,46}$ The slow and the fast modes correspond to the discrete modes whose phase speeds approach the phase speeds of the slow and the fast acoustic wave, $1-1 / M_{e}$ and $1+1 / M_{e}$, respectively, as the Reynolds number decreases towards zero. As the Reynolds number increases, the phase speed of the slow mode increases gradually from $1-1 / M_{e}$ to $C_{s}$ and the phase speed of the fast mode decreases gradually from $1+1 / M_{e}$ to 1.0 . As the phase speed of the fast mode approaches 1.0, the fast mode is absorbed into the continuous spectrum. Downstream of this point, a new mode with slightly different eigenvalues and eigenfunctions springs from the continuous spectrum. Even though there is a discontinuity in the eigenvalues and eigenfunctions across the continuous spectrum, the new mode is continued to be termed as the fast mode. Further downstream, the phase speed of the fast mode continues to decrease and at some point the phase speeds of the slow, and the fast modes cross each other. This point is termed as the synchronization point. However, the imaginary parts of the modes remain separate at this point.

Building on the fact that the phase speeds of the slow and fast acoustic waves synchronize with the phase speeds of the slow and fast modes, several investigations were conducted on the interaction of acoustic waves with supersonic and hypersonic boundary layers. Fedorov and Khokhlov ${ }^{47,48}$ showed using asymptotic theory that eigen solutions are formed near the leading-edge region of a flat plate during the diffraction of the acoustic wave by the growing boundary layer. This diffraction zone is very long in the streamwise direction on the order of $\varepsilon^{-2} \lambda$ in the streamwise direction, where $\varepsilon$ is an asymptotically small parameter and $\lambda$ is the wavelength of the free stream acoustic disturbances. This analysis is valid only in flows over very sharp geometries. Fedorov ${ }^{49}$ also identified that the unstable second mode can be generated through the inter-modal exchange between the fast and the slow modes near the synchronization point. For an adiabatic wall, it was also found that receptivity to slow acoustic waves could be as much as 50 times the receptivity to the fast acoustic waves ${ }^{49}$. Thus, the leading-edge receptivity via the slow mode excitation is much stronger than in the case of inter-modal exchange.

Ma and Zhong ${ }^{50,51}$ have performed a series of direct numerical simulations for a flow over a sharp Mach 4.5 flatplate boundary layer to investigate receptivity to fast acoustic waves. They used a fifth-order accurate shock-fitting method in the numerical simulation. They identified from the wavenumber and the eigenfucntions comparison with the linear stability calculations that the fast stable mode excited the unstable Mack's second mode through the mechanism of inter-modal exchange. They also considered the effects of incidence angles on the receptivity of the supersonic boundary layer to fast acoustic waves. The results showed that maximum receptivity of the second mode is obtained when the incident wave angle approximately equals 26. In a recent study, Egorov, Fedrov, and Soudakov $^{52}$ carried out a similar study at a Mach number of 6 whereby simulation of receptivity to slow and fast acoustic waves was performed and the effect of incidence angle was studied. Only the 'sharp' leading-edge case was considered. 
We have been investigating the stability and receptivity of hypersonic boundary layers to acoustic and vortical disturbances and have published the findings in several articles. The results for the interaction of plane acoustic waves with a flat plate (Malik and Balakumar ${ }^{53}$ ), a sharp and blunt cone (Kara and Balakumar ${ }^{54,55,56,57}$ ), and straight and flared cones (Balakumar and Kegerise ${ }^{58}$ ) were given in the referenced articles. The interactions of acoustic disturbances and vortical disturbances with a sharp wedge and a cone were documented in a paper by Balakumar and Kegerise. ${ }^{59}$ The receptivity of hypersonic boundary layers to distributed roughness and acoustic disturbances was studied by Balakumar. ${ }^{61}$ The stability of hypersonic boundary layers on a cone at an angle of attack and the generation of crossflow vortices from isolated roughness elements were investigated by Balakumar and Owens. ${ }^{60}$ In this paper we will summarize the findings for the receptivity of hypersonic boundary layers over wedges, cones, and flared cones to acoustic and vortical disturbances.

\section{Governing Equations}

The governing equations are the two-dimensional unsteady compressible Navier-Stokes equations, written in conservation form and in cylindrical coordinates:

$$
\begin{gathered}
\frac{\partial}{\partial t} r Q+\frac{\partial r F}{\partial x}+\frac{\partial r G}{\partial r}=S, \\
Q=\left[\begin{array}{l}
\rho \\
\rho E \\
\rho u \\
\rho v
\end{array}\right]
\end{gathered}
$$

Here, $(x, r)$ are the cylindrical coordinates, $(u, v)$ are the velocity components, $\rho$ is the density, and $p$ is the pressure. The total energy, $E$, is given by:

$$
\begin{gathered}
E=e+\frac{u^{2}+v^{2}}{2}, \\
e=c_{v} T, p=\rho R T,
\end{gathered}
$$

where $e$ is the internal energy and $T$ is the temperature. The fluxes $F, G$, and the source term $S$ are described in Kara et $a l{ }^{2}$ The viscosity, $\mu$, is computed using Sutherland's law and the thermal conductivity, $k$, is given in terms of the Prandtl number, $\operatorname{Pr}$. The variables $\rho, p, T$ and velocity are non-dimensionalized by their corresponding reference variables $\rho_{\infty}, p_{\infty}, T_{\infty}$ and $\sqrt{R T_{\infty}}$ respectively. The reference value for length is given by $\sqrt{v x_{0} / U_{\infty}}$, where $x_{0}$ is a reference location. For the computations, the equations were transformed from the physical coordinate system $(x, r)$ to the computational curvilinear coordinate system $(\xi, \eta)$ in a conservative manner.

\section{A. Solution Algorithm}

The governing equations were solved using a $5^{\text {th }}$-order accurate weighted essentially non-oscillatory (WENO) scheme for space discretization and a $3^{\text {rd }}$-order total-variation-diminishing (TVD) Runge-Kutta scheme for time integration. The WENO and TVD methods and formulas are explained in Shu. ${ }^{62}$ The application of the essentially non-oscillatory (ENO) method to the Navier-Stokes equations is presented by Atkins ${ }^{63}$. The solution method implemented in the present computations is described in Balakumar. ${ }^{64}$

A schematic diagram of the computational setup is shown in Fig. 1. Two-dimensional or axi-symmetric bodies are placed along the $x$-axis. The outer boundary of the computational domain lies outside the shock and follows a parabola. At the outflow boundary of the computational domain, an extrapolation boundary condition was used. At the wall, the simulations employed viscous conditions for the velocities and an adiabatic or constant wall temperature for the temperature. The density was computed from the continuity equation. In the mean-flow computations, the free-stream values at the outer boundary were prescribed. In the unsteady computations, acoustic 
or vortical perturbations were superimposed on the uniform mean flow at the outer boundary of the computational domain.

The solution procedure was to first compute the steady mean flow by performing unsteady computations using a variable time step until the maximum residual reaches a small value $\left(\sim 10^{-11}\right)$. The next steps were $(1)$ to introduce unsteady acoustic or vortical disturbances at the outer boundary of the computational domain and (2) to perform time accurate simulations to investigate the interaction of these disturbances with the boundary layer and their subsequent downstream evolution. Linearized Euler equations in a uniform mean flow are solved in Cartesian and cylindrical coordinates to obtain analytical expressions for the acoustic and vortical disturbances that were to be superimposed at the outer boundary ${ }^{60}$. Since we use a very fine spatial grid to resolve the leading-edge region, these computations require a very small time step, which is determined by the minimum time step allowed by the CFL number. The computational domain is divided into two regions and larger time steps can be taken in the downstream region. While the simulation allows nonlinear effects, the forcing amplitude is kept small enough in this study for dominant mechanisms to be governed by linear dynamics. The computational grid, which was generated using analytical formulae, stretches in the $\mathrm{h}$ direction close to the wall and is uniform outside of the boundary layer. In the $\mathrm{x}$ direction, the grid was very fine near the nose and then uniform in the region downstream. We generally use 251-501 points in the wall normal direction and about 25 points per wavelength in the streamwise direction.

\section{Results}

\section{A. Stability of Similarity Profiles for a $7^{\circ}$ Half-Angle Sharp-Tipped Cone}

First to orient the reader, we present the stability characteristics of a similarity hypersonic boundary layer over a sharp-tipped $7^{\circ}$ half-angle cone. Mack ${ }^{4}$ gave a wealth of information about the stability characteristics of compressible similarity profiles for a wide range of Mach numbers and wall conditions. We present the stability results for a freestream Mach number of $M=6$ and a freestream stagnation temperature of $430 \mathrm{~K}$. The inviscid conditions along the cone surface are obtained by solving the Taylor-Maccoll equation for conical flows. The shock angle is 11.94 degrees, the Mach number and the temperature along the surface of the cone are 5.355 and $63.84 \mathrm{~K}$, respectively. The stability results are presented for adiabatic wall conditions. The Prandtl number is assumed to be a constant value of 0.70 and the viscosity is calculated using the Sutherland law.

Figure 2 shows the mean density and velocity profiles obtained by solving the similarity equations. We plotted the variation of the generalized velocity derivative $\left(\rho U^{\prime}\right)$ and marked the sonic point and the generalized inflection point in the figure. At the sonic point, the mean velocity is $1-1 / M_{e}$ where $M_{e}$ is the boundary-layer edge Mach number. Relative to the mean velocity at the sonic point the velocity at the boundary-layer edge is sonic. The boundary-layer thickness in the similarity variable is $\delta_{999}=11.4$. The sonic point is located at $\eta=7.2$ and the generalized inflection point is located at $\eta=8.3$. The corresponding mean velocities at those points are 0.81 and 0.92. The phase velocities of the unstable disturbances are confined to this narrow region, about $1 / 10$ of the boundary-layer thickness, and this region is located between 65 to 73 percent of the boundary layer.

Figures 3-6 show typical linear stability diagrams for a hypersonic boundary layer. Figure 3(a) depicts the neutral stability curves in the nondimensional frequency, $F=2 \pi v_{e} f / U_{e}^{2}$, and the square root of the Reynolds number $\sqrt{\operatorname{Re}_{s}}=\sqrt{U_{e} s / v_{e}}$ plane and Fig. 3(b) depicts neutral curve in the phase speed and the Reynolds number plane. Figures 4(a) and (b) show the growth rates and the N-Factor curves for different frequencies. The neutral stability curve shows two lobes, one at high frequencies and the second at low frequencies. The high frequency unstable region is very narrow in the Reynolds number range shown and the low frequency unstable region is very long in the Reynolds number range shown. However, the growth rate curves for the lower frequencies show a very long almost neutral region at low Reynolds numbers before the growth rates grow to large values in a narrow region at high Reynolds numbers. The phase speeds of the instability waves are confined between $C_{0}=1-1 / M_{e}$ and $C_{s}$ where $C_{s}$ is the mean velocity at the generalized inflection point. The N-Factor of 10 occurs for a frequency of $F=0.80 * 10^{-4}$ and the square root of the Reynolds number at this location is $\sqrt{ } \mathrm{Re}_{\mathrm{s}}=3700$. Figure 5 shows the eigenfunction distributions for the pressure and mass-flux at two Reynolds numbers $\sqrt{ } \mathrm{Re}_{\mathrm{s}}=1100$ and 3000 for the frequency $F=0.80 * 10^{-4}$. These two points are marked in Fig. 3(a). The eigenfunctions are normalized by the pressure perturbations at the wall. It is seen that at the upstream neutral point $\sqrt{ } \mathrm{Re}_{\mathrm{s}}=1100$, the pressure peaks at the wall and remains almost constant inside the boundary layer before it decays gradually in the outer part and the mass-flux peaks near the critical layer with a magnitude about 4.5 times the pressure at the wall. At the downstream station, the pressure eigenfunction peaks at the wall and decays gradually inside the boundary layer and the mass-flux again peaks near the critical layer, but the amplitude is almost the same as the pressure at the wall. 
Figures 6(a) and 6(b) show the evolution of the eigenvalues along the axial direction for the slow (Mode S) and fast (Mode F) modes for the frequency $F=0.80 * 10^{-4}$. Figure 6(a) shows the evolution of phase speed $c_{r}$ and the growth rate $-\alpha_{i}$, and Fig. 6(b) shows the wavenumber $\alpha_{r}$. The slow mode or the Mode S synchronizes with the slow acoustic wave with the phase speed of $\left(U_{e}-a_{e}\right)$ or $1-1 / M_{e}$ as the Reynolds number or $x$ becomes small. The fast mode or the Mode F synchronizes with the fast acoustic wave with the phase speed of $\left(U_{e}+a_{e}\right)$ or $1+1 / M_{e}$ as the Reynolds number becomes small. The phase speed of the slow mode increases from $1-1 / M_{e}$ to $C_{s}$ as the Reynolds number increases while the phase speed of the fast mode decreases from $1+1 / M_{e}$ to a smaller value. As the fast mode evolves downstream, it merges with the continuous spectrum with phase speed $c_{r}=1.0$. For this frequency of $F=0.80 * 10^{-4}$ the merging occurs around $\sqrt{ } \operatorname{Re}_{\mathrm{s}}=2700$. Downstream of the continuous spectrum a new mode with slightly different eigenvalues and eigenfunctions emerges. These points are also marked as red dots in Fig. 6(a). Hence, the fast mode has a discontinuity across the continuous spectrum. This may have some effects in the receptivity process of generating the unstable second mode. As the slow mode and the new fast mode evolve downstream, the slow mode becomes the unstable Mack's second mode and the fast mode becomes the stable mode. Another observation is that the phase speeds of the slow mode and the fast mode coalesce near $\sqrt{ } \operatorname{Re}_{\mathrm{s}}=3120$. However, the growth rates of the slow mode and the fast mode at this point are quite different. The growth rates are $-\alpha_{i}=0.0064$ and -0.0166 for the slow and the fast modes, respectively. This point is also marked as a line in Figs 6 (a) and (b). Fedorov ${ }^{49}$ called this point the synchronization point for the fast and the slow mode and claimed that near this point inter-modal energy exchange could occur depending on the Mach number, frequency, and the wall conditions. He developed an analytical model using a quadratic dispersion relation to explain the observed growth rate pattern in hypersonic boundary layers. The important question is what happens when a fast mode evolves downstream? Does it generate an unstable second mode near the continuous spectrum point or near the synchronization point? We will discuss these questions in latter sections.

In the rest of the sections, we will concentrate on the receptivity process in hypersonic flows over several geometries including wedges, cones, and flared cones. Specifically, we will consider interactions of freestream acoustic and vortical disturbances with these geometries and investigate the generation and evolution of different instability modes inside the boundary layer.

\section{B. $7^{\circ}$ Half-Angle Sharp-Tipped Wedge}

We consider flow over a $7^{\circ}$ half-angle sharp-tipped wedge with a nose radius of $0.015 \mathrm{~mm}$. Computations were performed for a freestream Mach number of 6 . The freestream stagnation temperature and pressure were $430 \mathrm{~K}$ and $12.2 \mathrm{kPa}(140 \mathrm{psi})$, yielding a unit Reynolds number of $10.4 * 10^{6} \mathrm{~m}^{-1}$. The simulations were performed for a constant wall temperature of 300 K. Detailed results were given in Balakumar and Kegerise ${ }^{60}$.

\section{(1) Mean Flow and Linear Stability}

Figure 7 presents the computed mean density contours for the sharp-tipped wedge. The flow field for the entire computational domain is shown in Fig. 7, while the insert shows a close-up view of the nose region. The leadingedge shock is located approximately at $0.01 \mathrm{~mm}$ upstream of the leading edge. The density profiles at $x=1.0,3.0$, 10.0, 20.030 .0 and $50.0 \mathrm{~cm}$ are plotted in Fig. 8(a). Figure 8(b) shows the same profiles in the similarity coordinates. The compressible Blasius similarity profile is also included for comparison. The boundary-layer profiles slowly approach the Blasius similarity profile close to $x=50 \mathrm{~cm}$. The boundary-layer thickness near $x=50$ $\mathrm{cm}$ is about $3 \mathrm{~mm}$.

Linear stability results for the mean flow profiles obtained from the simulation revealed that the maximum NFactor reached within the computational domain of $x=50 \mathrm{~cm}$ is about 4.0 and the most amplified frequency is $F=$ $0.95 * 10^{-4}(137 \mathrm{kHz})$. The linear stability eigenvalue calculations showed that the fast mode merges with the continuous spectrum with phase speed $c_{r}=1.0$ around $x=281 \mathrm{~mm}$ and the phase speeds of the slow mode and the fast mode coalesce near $x=400 \mathrm{~mm}$.

\section{(2) Interaction of Acoustic Waves with the sharp-tipped wedge}

After the mean flow was computed, two-dimensional acoustic disturbances at different incidence angles were introduced at the outer boundary of the computational domain and time-accurate simulations were performed. Computations were performed with the slow and fast acoustic waves at 0 degrees incidence angle and were performed with the slow wave for the other incidence angles. The amplitude of these forced acoustic disturbances was given a small value of $\tilde{p}_{a c} / p_{\infty}=1 * 10^{-5}$ to ensure that the disturbances evolving in the boundary layer remained in the linear regime. We discuss the results for a frequency of $F=0.95^{*} 10^{-4}(137 \mathrm{kHz})$. Figures 9 (a-d) show the unsteady density fluctuations at a fixed time for the acoustic incidence angle of 0 and 20 degrees obtained with the slow acoustic wave and Figs. 9(e, f) show the results for the 0 deg with the fast acoustic wave. The results for other 
incidence angles of 10 and 30 degrees are in Ref. 60. The observations are similar to our previous computational results ${ }^{53}$ for the flow over a flat plate at a supersonic Mach number of 4.5. The perturbation field can be divided into four regions. One region is the area outside the shock where the acoustic waves propagate uniformly. The second region is the shock layer across which the acoustic waves are transmitted. The third region is the area between the shock and the boundary layer. This region consists of the transmitted external acoustic field and the disturbances that are radiated from the boundary layer. The fourth region is the boundary layer where the boundary-layer disturbances evolve. It is seen that at zero incidence angle for the slow mode, the acoustic waves are very weakly transmitted through the shock. It is also observed that the fluctuations inside the boundary layer are larger than the amplitude of the incoming acoustic field. It suggests that the boundary layers are perturbed strongly starting from the nose region. With increasing incidence angles, it is noted that the acoustic waves are still weakly transmitted through the shock in the lower part of the domain while they are strongly transmitted through the shock in the upper part as evident from the disturbed flow field between the wedge surface and the shock. Another observation is that the fluctuations inside the boundary layer in the lower part are lower than that in the upper part. Figures 9(e, f) display the density fluctuations induced by the fast mode at 0 degrees incidence angle. It is seen that the fast acoustic mode is transmitted strongly across the shock compared to the slow mode case, Figs. $9(\mathrm{a}, \mathrm{b})$. The perturbations inside the boundary layer are larger than the amplitude of the incoming wave.

Figures 10(a-b) show the amplitude of the pressure fluctuations at the lower and upper side of the wall for the incident angles of 0 and 20 degrees. Figure 10 also includes the results from the parabolized stability equations (PSE) computations obtained for the same mean boundary-layer profiles. The growth of the disturbances agrees very well with the PSE results a short distance downstream of the leading edge. Figure 10(a) shows the results for the slow and the fast modes at zero incidence angles. The figure for the slow wave clearly shows the initial generation and the eventual exponential growth of the instability waves inside the boundary layer. The slow acoustic wave whose wavelength $(5.29 \mathrm{~mm})$ is close to the wavelength $(5.16 \mathrm{~mm})$ of the slow mode (Mode $\mathrm{S}$ ) near the leading edge transforms into instability waves rather directly. Similarly, the fast acoustic mode whose wavelength (7.41 $\mathrm{mm}$ ) is close to the wavelength $(7.41 \mathrm{~mm})$ of the fast mode (Mode F) near the leading edge synchronizes with the instability mode near the leading-edge region. Figure 10(a) also illustrates that as the disturbance induced by the fast mode evolves downstream, it first grows up to $x \sim 12 \mathrm{~cm}$. and then decreases to a smaller value near $x \sim 34 \mathrm{~cm}$. before it grows again due to the second mode instability. As we discussed earlier, the stable fast mode generated the unstable second mode near the continuous spectrum $(x=28 \mathrm{~cm}$.) or near the synchronization point $(x=40 \mathrm{~cm}$.). However, the unstable second mode generated by the fast mode is weak compared to that generated by the slow mode. The maximum amplitude reached in the slow acoustic wave case is about 0.00154 and it is about 0.00044 in the fast acoustic case. Hence, the slow mode is about four times more efficient in generating the unstable mode compared to the fast mode. From these values the receptivity coefficients defined by the initial amplitude of the pressure fluctuations at the wall non-dimensionalized by the free-stream acoustic pressure can be evaluated.

$$
C_{\text {recpt, }, p_{\text {wall }}}=\frac{\left(p_{\text {wall }}\right)_{n}}{p_{a c}}
$$

The computed receptivity coefficient associated with the slow acoustic wave is about

$$
C_{\text {recpt, } p_{\text {wall }}, S}=2.5
$$

Figure 10(b) shows the pressure fluctuations at the upper and the lower walls for incidence angle of 20 degrees. We can see that the maximum amplitudes are smaller on the lower (windward) side compared to the upper (leeward) side. This effect increases with increasing angles of incidence. The maximum amplitudes of the pressure fluctuations reached on the upper wall for the different incidence angles $0,10,20$, and 30 degrees are $0.0015,0.0023,0.0027$, and 0.0023 , respectively. The maximum amplitudes reached on the lower walls are $0.0015,0.00093,0.00058$, 0.00038 , respectively. Hence the receptivity becomes weaker on the lower side compared to the upper side with increasing incidence angles. The maximum amplitude on the upper surface increased by a factor of 1.8 for 20 degrees incidence angle and then decreases slowly for larger incidence angles.

To see how the wavenumber of the fluctuations inside the boundary layer varies along the streamwise direction and how they compare with the wavenumber obtained from the linear stability computations, the wavenumber of the pressure fluctuations along the wall is computed. This is achieved by first decomposing the fluctuations into harmonic components of the form

$$
p(x, t)=\tilde{p}(x) e^{-i \omega t}+c . c
$$

and then evaluating the wavenumber of the fluctuations from the expression 


$$
\begin{aligned}
\alpha(x) & =\left(\frac{1}{i \tilde{p}(x)} \frac{\partial \tilde{p}(x)}{\partial x}\right) \\
& =\alpha_{r}+i \alpha_{i}
\end{aligned}
$$

Figure 11 shows the computed wavenumber variation along the streamwise direction. Figure 11(a) gives the results for both the slow and fast acoustic waves cases while Fig. 11(b) gives the results for the 20 degrees incidence angle case. The figures also include the wavenumber distributions obtained from linear stability computations for the slow and the fast modes. Downstream of the leading-edge region $x>10.0 \mathrm{~mm}$, the wavenumber distributions computed using Eq. (6) agree very well with the linear stability theory results in all the cases. The wavenumber variations very near the nose region are shown in the insert in Fig. 11(a). The wavenumber first decreases up to $x \sim 1$ $\mathrm{mm}$, it then increases slowly for $x>1 \mathrm{~mm}$ and merges with the slow and the fast instability modes around $x \sim 20$ $\mathrm{mm}$. The two solutions, from the simulation and the linear stability calculations, continue to agree beyond this point for the slow modes. The fast stability mode is also excited very near the leading edge as is evident by the agreement between the linear stability and the numerical simulation results. At a location close to $x=200 \mathrm{~mm}$, the wavenumber starts to oscillate and merges with the values corresponding to the slow mode around $x \sim 400 \mathrm{~mm}$. This is the region where the energy is transferred to the slow mode from the fast mode. The locations corresponding to the continuous spectrum and to the synchronization point are also marked in the figure.

\section{(3) Interaction of Vorticity Waves with the sharp-tipped wedge}

Figures 12(a-d) show the density and the vortical velocity, $v$, perturbations generated by the interaction of vortical disturbances with the boundary layer. Since vortical disturbances do not contain density or pressure fluctuations in the free stream, density fluctuations appear only between the shock and the wall due to the interaction of vortical disturbances with the shock. The flow field revealed by the velocity perturbations can be divided, as discussed in the previous section, into four regions. One is the region outside the shock where the perturbations are uniform and governed by the linerized Euler equations. The second is the region between the shock and the boundary layer away from the leading edge. Here, the incoming vortical wave is diffracted towards the normal to the shock and the amplitude of the wave is increased across the shock. However, the diffracted waves do not penetrate the boundary layer. The third is the region between the shock and the wall near the leading edge. Here, the incoming vortical disturbances are diffracted and elongated and the amplitude is decreased across the shock. The fourth is the boundary-layer region. Figures 12(c) and (d) show that the perturbations inside the boundary layer are generated starting from the leading edge.

Figure 13 depicts the wall pressure fluctuations generated by the interaction of vortical disturbances with the wedge. The results show that up to $x \sim 28 \mathrm{~cm}$, the oscillations consist of short wavelength disturbances that are modulated by long wavelength disturbances. Beyond $x \sim 28 \mathrm{~cm}$, the short wavelength disturbances merge into the unstable second mode wave. Figure 14 compares the wall pressure oscillations generated by the slow and fast acoustic wave and by the vortical disturbance. The maximum amplitudes generated by the interaction with these waves are $0.0015,0.00044$ and 0.00035 , respectively. Hence the fast acoustic wave is about 3.4 times less efficient than the slow acoustic wave, and the vorticity wave is about 4.2 times less efficient in generating the unstable second mode in an adiabatic boundary layer over a wedge. These numbers may change with frequencies, however, the conclusions will remain the same.

Figure 15 shows the calculated wavenumber variation for this case. The wavenumbers computed from the linear instability are also included in the figure for comparison. It is interesting to see that the wavenumber of the fluctuations follows the wavenumber for the fast instability mode up to $x \sim 250 \mathrm{~mm}$ and jumps to the wavenumber for the slow instability mode near $x \sim 280 \mathrm{~mm}$. This is the location where the fast mode is absorbed into the continuous spectrum. Hence the switching or the transformation from the fast instability mode to the slow instability mode in this case occurs near the continuous spectrum.

\section{C. $7^{\circ}$ Half-Angle Sharp-Tipped Cone}

Similar to the wedge case, in this section, we discuss the results for the flow over a sharp-tipped cone. Figures 16(a) and (b) show the computed mean density contours for the sharp-tipped cone. The freestream conditions are the same as that for the wedge. This cone model geometry was tested in the Boeing/AFOSR Mach 6 Quiet Tunnel at the same freestream conditions. ${ }^{15}$ The mean flow boundary-layer profiles and linear-stability results were given in Ref. 60 . The boundary-layer profiles slowly approach the Blasius similarity profile by $x=3.0 \mathrm{~cm}$. The computed Nfactor curves obtained from the linear-stability analysis and the PSE calculations are plotted in Fig. 17. For the PSE results in particular, the presented N-Factors were obtained using $(\rho u)_{\max }$. The two axial locations annotated in the 
figure at $x=208$ and $490 \mathrm{~mm}$, correspond to those where measured wall-pressure fluctuation data are available for comparison. ${ }^{15}$ At these locations, the maximum N-factors obtained from the linear stability computations are 3.1 and 6.5, respectively, and the corresponding frequencies are 350 and $220 \mathrm{kHz}$. The corresponding $\mathrm{N}$-factors obtained from the PSE computations are 4.4 and 7.7, respectively. These increases in the N-factors in the PSE computations are largely due to the growth of the disturbances in the first-mode region. The $\mathrm{N}$-factors at the neutral points $(x=10$ and $26 \mathrm{~cm})$ for the frequencies 350 and $220 \mathrm{kHz}$ are 0.8 and 1.2, respectively. Hence, due to the nonparallel effects, the first mode becomes unstable near the nose region of the cone. The continuous spectrum is located around $x=281 \mathrm{~mm}$ and the synchronization of the phase speeds for the slow and the fast modes occurs near $x=360 \mathrm{~mm}$.

\section{(1) Interaction of Slow and Fast Acoustic Waves with the cone}

As in the previous case, after the mean flow was computed, two-dimensional slow and fast acoustic disturbances (with an amplitude of $\left.\tilde{p}_{a c} / p_{\infty}=1 * 10^{-5}\right)$ at an incidence angle of 0 degrees at a frequency of $F=1.5^{*} 10^{-4}(217 \mathrm{kHz})$ were introduced at the outer boundary of the computational domain and time-accurate simulations were performed. Contours of the density fluctuations generated by the slow and fast acoustic waves are shown in Figs. 18(a-d). The flow features are similar to the wedge case. For the slow mode, the acoustic waves are very weakly transmitted through the shock. It is also observed that the fluctuations inside the boundary layer are larger than the amplitude of the incoming acoustic field. This suggests that the boundary layer is perturbed strongly starting from the nose region. The fast acoustic mode is transmitted strongly across the shock as compared to the slow mode case. The perturbations inside the boundary layer are larger than the amplitude of the incoming wave. Fig. 18(d) also shows that the amplitude inside the boundary layer does not increase or decrease uniformly. In some regions, it increases and in some regions, it decreases to very small values.

Figures 19(a-b) show the amplitude of the pressure fluctuations along the surface of the cone for the slow and the fast acoustic waves. Figure 19 also includes the results from the parabolized stability equations (PSE) computations obtained for the same mean boundary-layer profiles. The PSE calculations are started at $x=0.45 \mathrm{~cm}$ with the initial conditions obtained from the eigenfunctions obtained from the linear stability calculations. The figure for the slow wave clearly shows the initial generation and the eventual exponential growth of the instability waves inside the boundary layer. The slow acoustic wave whose wavelength $(3.35 \mathrm{~mm})$ is close to the wavelength of the slow mode (Mode S) near the leading edge $(3.47 \mathrm{~mm})$ transforms into instability waves rather directly. Similarly, the fast acoustic mode whose wavelength $(4.69 \mathrm{~mm})$ is close to the wavelength of the fast mode (Mode F) near the leadingedge $(4.64 \mathrm{~mm})$ synchronizes with the instability mode near the leading edge region. As was observed in Fig. 10(a), the fast mode does not evolve into an unstable second mode as the Reynolds number increases. Figure 19(b) shows that as the disturbance induced by the fast mode evolves downstream, it first grows up to $x \sim 12 \mathrm{~cm}$, then decreases and oscillates before it grows due to the second mode instability. The unstable second mode generated by the fast mode is weak compared to that generated by the slow mode. The maximum amplitude reached in the slow acoustic wave case is about 0.213 and it is about 0.0023 in the fast acoustic case. Hence, the slow mode is about ninety times more efficient in generating the unstable mode compared to the fast mode for this sharp-tipped cone.

Figure 20 shows the computed wavenumber variation along the streamwise direction for the slow and fast acoustic wave cases. The figures also include the wavenumber distributions obtained from linear stability computations for the slow and the fast instability modes. It is seen that the wavenumbers obtained from the DNS and the linear stability merge starting from $x \sim 10 \mathrm{~mm}$ for the slow wave and from $x \sim 2 \mathrm{~mm}$ for the fast wave. Hence, if we consider $x \sim 10 \mathrm{~mm}$ as the end of the receptivity process for the slow wave, the simulation suggests that the receptivity region extends for about three wavelengths from the nose. The agreement between the PSE and the DNS is also further evidence that the boundary-layer disturbances originate from a small region near the cone tip since the PSE calculations were performed with a disturbance input at a single axial location. The fast stability mode is also excited very near the leading edge as is evident by the agreement between the linear stability and the numerical simulation results. At a location close to $x=120 \mathrm{~mm}$, the wavenumber starts to oscillate and shifts to a lower value of 0.13 . Further downstream around $\mathrm{x} \sim 380 \mathrm{~mm}$, the wavenumber jumps to the value corresponding to the slow wave. This is the region where the energy is transferred from the fast mode to the slow mode. The amplitudes of the wall-pressure fluctuation for the slow mode at $x=6$ and $26 \mathrm{~cm}$ are $5.8 * 10^{-5}$ and $2.0^{*} 10^{-4}$, respectively. The first location is the neutral point from the PSE results and the second location is the neutral point from the linear stability results. With those amplitudes, and a freestream acoustic pressure level of $2 * 10^{-5}$, the receptivity coefficients are approximately 3 and 10 at those neutral points for a frequency of $216 \mathrm{kHz}$.

Figure 21 presents a comparison between the computed and the measured amplitudes of the wall-pressure fluctuations at two axial locations $(x=208$ and $490 \mathrm{~mm})$. Recall that the simulations were performed for freestream acoustic disturbances over a range of frequencies, so the amplitude data are presented as a function of frequency. 
Also in the computations, the amplitude of the acoustic disturbances was set to $\tilde{p}_{a c} / p_{\infty}=1 * 10^{-5}$ for all the frequencies. The actual amplitude of the freestream acoustic disturbances present in the experimental measurements is unknown. Figure 21(a) compares the computed wall-pressure amplitude at $x=208 \mathrm{~mm}, R e=10.4 * 10^{6} \mathrm{~m}^{-1}$ and the wall-pressure amplitude spectrum measured at the same axial location and flow condition by Casper et al. ${ }^{15}$ Note that the pressure spectrum shown in Fig. 21(a) was measured under noisy-flow conditions because under quietflow conditions, the wall-pressure fluctuations were below the noise floor of the pressure transducer. Also, the transducer used for this measurement had a sensor size of approximately $1 \mathrm{~mm}$, which is on the order of the instability wavelengths $(2.4$ to $3.5 \mathrm{~mm})$ in the boundary layer. Since the effect of spatial averaging on the sensor dynamic response was not characterized in the experiment, the measured spectrum amplitude shown in the plot is qualitative. Hence to compare with the experiments, we shifted the scales in the right axis to match the maximum amplitudes in the experiments and computations. Nevertheless, the frequencies and shape of the computed amplitude spectrum compare favorably with the experimental data. There is a small shift in the measured and computed frequencies near the maximum amplitudes. The measured frequency at the peak amplitude is about 340 $\mathrm{kHz}$ and the computed frequency is about $330 \mathrm{kHz}$. Figure 21(b) presents a similar comparison between the computational and experimental wall-pressure spectrum at $x=490 \mathrm{~mm}, R e=10.4 * 10^{6} \mathrm{~m}^{-1}$. This experimental data was obtained under quiet-flow conditions with the same pressure transducer. As before, the frequencies and shapes of the computed wall-pressure spectrum agree favorably with the measurements. The measured frequency at the peak amplitude is about $230 \mathrm{kHz}$ and the computed frequency is about $215 \mathrm{kHz}$. Although the spectral comparisons made here are qualitative in amplitude, the computational approach presented in this paper should be able to quantitatively predict the amplitude of the instability waves provided we know the actual amplitude of the freestream acoustic disturbances that were present in the experiment.

\section{(2) Interaction of Vorticity Waves with the sharp-tipped cone}

Figures 22(a-d) show the contours of the density and the axial velocity, $u$, perturbations generated by the interaction of vortical disturbances with the boundary layer. Figures 22(a) and (b) display the density fluctuations for the nose region and in the middle of the domain $x=31$ to $34 \mathrm{~cm}$, respectively. Figures 22(c) and (d) similarly depict the fluctuations for the axial velocity component. Since vortical disturbances do not contain density or pressure fluctuations in the free stream, density fluctuations appear only between the shock and the wall due to the interaction of vortical disturbances with the shock. The flow field revealed by the velocity perturbations are similar to that observed in the wedge case.

Figure 23 depicts the wall pressure fluctuations generated by the interaction of vortical disturbances with the cone. The results show that the disturbances grow up to $x \sim 30 \mathrm{~cm}$ then decrease to a minimum value close to $x=33$ $\mathrm{cm}$ and increase downstream due to the second mode instability. The maximum amplitude obtained in this case is about 0.12 . The fast mode is about 93 times less efficient than the slow mode, the axi-symmetric mode at an incidence wavenumber of $\alpha_{n}=0.05$ is about 1.25 times more efficient and the vortical disturbance is about 2.7 times less efficient

Figure 24 shows the calculated wavenumber variation for this case. The wavenumbers computed from the linear instability are also included in the figure for comparison. The wave number oscillates around 0.155 until $x \sim 300$ $\mathrm{mm}$, then increases slowly and merges with the wavenumber for the slow mode instability. It is observed that the wavenumber in the freestream for the vorticity wave is also about 0.153 . This suggests that the disturbances in the boundary layer up to $x \sim 300 \mathrm{~mm}$ are dominated by the transmitted vorticity waves. It is also noticed that the large oscillations in the wavenumber and the minimum amplitude in the pressure (Fig. 23) occur near the same location around $x \sim 330 \mathrm{~mm}$. At this point, we do not know the significance of this coincidence, however, it may be a hint in identifying the mechanism for generating the unstable second mode in this case. It would be interesting to compare the shape of the amplitude distributions with the eigenfunctions for the slow and the fast modes.

\section{D. $5^{\circ}$ Half-Angle Blunt Cone}

Computations were performed for hypersonic flows at a free stream Mach number of 6.0 over a 5-degree halfangle cone with spherical blunt noses with the nose radii of $r_{0}=0.001,0.01,0.05$ and 0.10 inches at a unit Reynolds number of $7.8^{*} 10^{6} / \mathrm{ft}$. This provides nose Reynolds numbers of $650,6500,32,500$, and 65,000 . To achieve higher nose Reynolds numbers, two computations were performed at a higher unit Reynolds number of $15.6 * 10^{6} / \mathrm{ft}$ for the nose radii of $r_{0}=0.05$ and 0.10 inches. The flow conditions and geometry are the same as that used in the experiments by Horvath et al. ${ }^{32}$ Detailed simulation results for different cases are given in Ref. $(55,57)$. 
Figures 25(a) and (b) depict the density and entropy contours for the bluntness case with nose radius of $r_{0}=0.10$ inches and a unit Reynolds number of $7.8 * 10^{6} / \mathrm{ft}$. For this large nose radius, the entropy layer that was visible near the nose region persisted downstream and eventually merged with the boundary layer. Figures 26(a) and (b) show the entropy profiles at different axial locations for the small and large nose radii of 0.001 and 0.10 inches respectively. For the small nose radius, only the boundary layer appears near the nose region and the entropy layer is not discernable in the outer part of the boundary layer. For the large nose radius, $r_{0}=0.10$ inches, two layers are clearly seen (marked by BL and EL in Fig. 26(b)) in the profiles at $x=0.50$ and 1.0 inches. One layer is very close to the wall with a large gradient and the other is away from the wall with a small gradient. The outside entropy layer merges with the boundary layer near the wall close to $x=10.0$ inches. The entropy layer and the boundary layer in non-dimensional units merge approximately 100 200 nose radii downstream from the nose.

\section{(1) Linear Stability}

Figures 27(a) and (b) show the N-Factors and the growth rates for the most amplified disturbances. They were computed using the mean profiles obtained from the numerical simulations for different bluntness at $r_{0}=0.001,0.01$, $0.05,0.10$ inches with the unit Reynolds number of $7.8^{*} 10^{6} / \mathrm{ft}$ and for $r_{0}=0.05,0.10$ inches with the higher unit Reynolds number of $15.6^{*} 10^{6} / \mathrm{ft}$. For comparison, the results for the Blasius similarity profiles, which model a sharp leading edge, are also shown in the figure. The non-dimensional frequency of the most amplified wave is about $F=$ $0.91 * 10^{-4}$ for the similarity profiles. The non-dimensional frequency $F=1.0 * 10^{-4}$ is equivalent to $390 \mathrm{kHz}$. There is a significant difference both for the mean flow and the stability results with increasing bluntness. The growth rates become smaller and the N-Factor curves move downstream with increasing bluntness. For the smaller bluntness of $r_{0}=0.001,0.01$ inch cases, the N-Factor curves remain closer to the similarity curve. For the larger bluntness cases, the growth rates become smaller and the N-Factor curves move further to the right. The most amplified frequencies are $\left(0.88^{*} 10^{-4}, 0.87 * 10^{-4}, 0.75^{*} 10^{-4}, 0.59^{*} 10^{-4}, 0.42 * 10^{-4}\right)$ for $r_{0}=0.001,0.01,0.05,0.10$ and 0.10 inches (with higher unit Reynolds number), respectively. This shows that the frequencies of the most amplified disturbances become smaller with increasing bluntness. The growth rate curves are similar to the Blasius profile for all the cases, with the exception that the mildly unstable first mode becomes stable. The transition Reynolds numbers obtained using the $\mathrm{N}$-factor of 10.0 for different bluntness cases are plotted in Fig. 28. The ratio between the transition Reynolds number with bluntness and the transition Reynolds number for the similarity profile, $\left(\operatorname{Re}_{\mathrm{T}}\right)_{\mathrm{r}} /\left(\mathrm{Re}_{\mathrm{T}}\right)_{\text {Similarity }}$, is about 1.06, 1.08, 1.37, 1.97, and 3.48, respectively for $\mathrm{Re}_{\mathrm{r} 0}=650,6500,32500,65000$, and 130000. To differentiate between the effects of free stream unit Reynolds number and the nose Reynolds number, one simulation was performed for the bluntness case $r_{0}=0.05$ at twice the unit Reynolds number of $2 * 7.8 * 10^{6} / \mathrm{ft}$. The expectation was that the results from this simulation would be close to the results obtained for the case with $r_{0}=0.10$ inch at a unit Reynolds number of $7.8^{*} 10^{6} / \mathrm{ft}$. It is interesting to see in Fig. 27 that the growth rates and the N-Factor curves are almost the same and the most amplified non-dimensional frequencies are also the same at $F=0.59 * 10^{-4}$. The transition Reynolds numbers are the same, $23.2 * 10^{6}$, for the cases of $r_{0}=0.10$ and 0.05 inches (higher unit Reynolds number), respectively. The transition Reynolds number for this case is also included in Fig. (28). This implies that the nose Reynolds number determines the stability and the transition of boundary layers over blunt bodies. Previous experiments ${ }^{20}$ and the stability calculations ${ }^{22}$ showed that the transition Reynolds number for a blunt cone at a Mach number of 8 , with nose Reynolds numbers of 30,000, increased by a factor of 1.7 2.0 compared to a sharp cone. Potter $^{33}$ found from a series of aeroballistics range experiments on nominally sharp cones that the transition Reynolds number increases with the free stream unit Reynolds number as a power of 0.63 . A line with the slope of 0.60 is included in Fig. (28) for comparison. The prediction from the present calculations follows this slope closely. Figure 29 shows the evolution of the eigenvalues, wavenumber $\alpha_{r}$ and growth rate $-\alpha_{i}$, along the axial direction for the slow (Mode S) and fast (Mode F) modes for the frequency $F=0.75^{*} 10^{-4}$ and $r_{0}=0.05$ inches. The continuous spectrum is located around $x=12$ inches and the synchronization of the phase speeds for the slow and the fast modes occurs near $x=17$ inches. Another observation is that compared to the sharp cone cases (Fig. 6), the first mode is strongly stabilized near the nose region.

\section{(2) Interaction of Acoustic Waves with the Boundary Layer}

Unsteady simulation results are discussed here for the cases of $r_{0}=0.001$ and 0.05 inches at the most amplified frequencies of $F=0.80 \times 10^{-4}$ and $0.75 \times 10^{-4}$. These frequencies correspond to 312 and $292 \mathrm{kHz}$, respectively. The amplitude of the forcing freestream acoustic waves was given a small value of $p_{a c} / p_{\infty}=2 * 10^{-6}$. Even with this small amplitude, nonlinearity started to develop near the end of computational domain for the small bluntness case of $r_{0}=0.001$ with a frequency of $F=0.80 \times 10^{-4}$.

Figures 30(a) and (b) show the density fluctuations inside the boundary layer near the nose region for a small $r_{0}=0.001$ inch and large $r_{0}=0.10$ inch cases (not to scale). We also included the shock and the edge of the entropy 
layer in these figures for illustration. The results for the small bluntness case are similar to the previous 7-deg halfangle cone case, Figs. 18 and 19. The Figs. 30(a) and (b) clearly distinguish the effect of bluntness in the generation of disturbances near the nose region. As was discussed previously for the small bluntness case, there was no entropy layer and the disturbances excited the boundary layer up to the wall. In the large bluntness case, the acoustic disturbances propagate across the leading-edge bow shock and perturb the entropy layer. These disturbances, as they evolve downstream, remain inside the entropy layer and get into the boundary layer further downstream.

Figures 31(a)-(c) show the evolution of the wall pressure fluctuations for these cases. These figures also include the results from the parabolized stability equations (PSE) computations obtained for the same mean boundary-layer profiles. The growth of the disturbances agrees very well with the PSE results. The figures clearly show the generation and the eventual exponential growth of the instability waves inside the boundary layer. The first observation is that there are large differences in the amplitude levels of the disturbances attained between the small bluntness case and the large bluntness cases. In all of the cases, the amplitude of the free stream acoustic pressure is the same, where $p_{a c} / p_{\infty}=2 * 10^{-6}$. For the nose radius, $r_{0}=0.001$ inch, Fig. 31(a), the disturbances grow from the leading edge and reach large amplitude levels of 0.50 near the predicted transition onset point. The maximum amplitudes obtained for the large nose radius, $r_{0}=0.05$ inch, are very small in the range of $\sim 10^{-4}$. The reason for this is the disappearance of the amplification of the first modes in the early part of the evolution. The disturbances not only grow, but decay by two orders in magnitude before they start to grow due to the instability of second modes. This may be due to the thickening of the boundary layer due to the entropy layer.

Following the PSE results up to the neutral point, the initial amplitude of the instability waves at the neutral point can be estimated. From these values, the receptivity coefficients defined by the amplitude of the pressure fluctuations at the wall, at the neutral point non-dimensionalized by the free-stream acoustic pressure, can be evaluated:

$$
C_{\text {recpt, }, p_{\text {wall }}}=\frac{\left(p_{\text {wall }}\right)_{n}}{p_{a c}}
$$

Table 1 shows the amplitude of the pressure fluctuations, $\left(p_{\text {wall }}\right)_{n}$, at the neutral point and the receptivity coefficients for different nose radii. The amplitudes near the neutral points are about $8.5 * 10^{-6}$ for the small bluntness case and they are on the order of $10^{-9}$ for the large bluntness cases. The receptivity coefficients are $4.23,1.85^{*} 10^{-3}$, and $4.75^{*} 10^{-3}$ for the cases of $r_{0}=0.001,0.05$, and 0.10 inches, respectively. Hence the receptivity coefficients are about 500-1000 times smaller for flows over blunt cones compared to that for the sharp cone. This is equivalent to an N-Factor of 6 to 7. This implies that the N-Factors at the transition onset in flows over blunt cones will be about 6 to 7 larger than that for the sharp cone. Another interesting observation can be made when comparing the amplitudes and the receptivity coefficients for the two cases of $r_{0}=0.10$ inch and $r_{0}=0.05$ inch $\left(2 * \operatorname{Re}_{\infty}\right)$; the amplitudes and the receptivity coefficients are almost the same for these two cases. Not only for the instability behavior, but the receptivity coefficients also appear to depend only on the nose Reynolds number. This implies that unit Reynolds number effect is directly the consequence of the variation in the nose Reynolds numbers.

Table 1 Receptivity coefficients for different nose radii

\begin{tabular}{ccccc}
\hline \hline$r_{0}$, in & $\mathrm{Re}_{\mathrm{r} 0}$ & $\mathrm{~F} \times 10^{-4}$ & $\left(p_{\text {wall }}\right)_{n}$ & $C_{\text {recpt }, p_{\text {wall }} \text {, Slow }}$ \\
\hline $0.001^{\&}$ & 650 & 0.80 & $8.5^{*} 10^{-6}$ & 4.23 \\
$0.050^{\&}$ & 32,500 & 0.75 & $3.7^{*} 10^{-9}$ & $1.85^{*} 10^{-3}$ \\
$0.100^{\&}$ & 65,000 & 0.60 & $9.5^{*} 10^{-9}$ & $4.75^{*} 10^{-3}$ \\
$0.050^{\#}$ & 65,000 & 0.60 & $10.0^{*} 10^{-9}$ & $5.00^{*} 10^{-3}$ \\
\hline \hline
\end{tabular}

${ }^{\&} \mathrm{Re}_{\infty}=7.8^{*} 10^{6},{ }^{\#} \mathrm{Re}_{\infty}=15.6 * 10^{6}$

Figure 32 shows the computed wavenumber variation along the streamwise direction for the slow and fast acoustic wave cases for the frequency $F=0.75 * 10^{-4}$ and $r_{0}=0.05$ inches. The figure also includes the wavenumber distributions obtained from linear stability computations for the slow and the fast instability modes, the wavenumber variations for the slow acoustic, fast acoustic, and vorticity waves based on the boundary-layer edge conditions. The first observation is that near the nose region the slow and the fast acoustic waves generate only the fast instability mode. Near $x \sim 6$ inches, the wavenumber for the slow wave switches to the wavenumber vorticity wave and the wavenumber for the fast wave switches to the fast acoustic wave. Further downstream, the wavenumbers of the disturbances generated by the slow acoustic wave oscillate around the wavenumber for the vorticity waves. The wavenumbers of the disturbances generated by the fast wave closely follows the wavenumber for the fast acoustic 
wave. Close to $x=17$ inches, the wavenumber distribution for the disturbances generated by the slow acoustic wave jumps to the wavenumbers corresponding to the unstable second mode wavenumber obtained from the linear stability theory. It is to be noted that this location corresponds to the synchronization point between the slow and the fast instability modes. Similarly the wavenumber distribution for the disturbances generated by the fast acoustic wave jumps to the wavenumber distributions for the second mode close to $x=22$ inches. The important conclusion is that the unstable second mode is generated only in the downstream part of the blunt cone. We do not know at this time the reasons or the mechanisms that caused these transformations to the unstable second mode at these specific locations and will explore them in future using modal analysis employing adjoint techniques.

\section{E. $5^{\circ}$ Half-Angle Sharp-Tipped Cone with Cold Wall}

Computations were performed for hypersonic flow at a free stream Mach number of 6.0 over a 5-degree halfangle cone with a sharp leading edge with radius $r_{0}=0.001$ inches, for different wall temperatures to investigate the effects of wall cooling on hypersonic boundary-layer receptivity due to acoustic disturbances and on stability. The flow parameters are the same as in the previous section D. Simulations were performed for wall temperatures $T_{w}=$ adiabatic wall $\left(T_{a w}\right), 0.75 * T_{a w}, 0.50 * T_{a w}, 0.40 * T_{a w}, 0.30 * T_{a w}$, and $0.20 * T_{a w}$. Mean flow profiles, linear stability, and receptivity results for all the cases were given in Ref. (56). We summarize the findings in this section for the case $T_{w}=0.20 * T_{a w}$.

\section{(1) Linear stability}

Due to the wall cooling, the boundary-layer thickness becomes smaller compared to the adiabatic case and the frequencies of the most amplified waves increase. The wall cooling stabilizes the first mode and destabilizes the second mode. Figure 33(a) depicts the growth rates and the wavnumbers for the slow and fast modes for a frequency of $F=1.7^{*} 10^{-4}(663 \mathrm{kHz})$. The continuous spectrum is located at $x=5.6$ inches and the wavenumbers for the slow mode and the fast mode coalesce starting at $x=6.33$ inches and remain almost the same until $x=10.3$ inches. However, the growth rates for the slow and the fast waves remain vastly separated in this region. Figure 33(b) shows the N-Factor variations for the adiabatic and cold wall cases. The most amplified frequencies that reach N-Factor values of 10 are $F=0.88 * 10^{-4}$ and $1.7 * 10^{-4}$ for the adiabatic and cold wall cases, respectively. The N-Factor of 10 occurs at $x=19.5$ and 9.5 inches for the adiabatic and cold wall cases, respectively. Hence, based on the local instability analysis the cold wall moves the transition onset farther upstream compared to the adiabatic wall case.

\section{(2) Interaction of Slow and Fast Acoustic Waves with the Boundary Layer}

Two-dimensional slow and fast acoustic disturbances were introduced at the outer computational boundary and the time accurate simulations were performed. Figure 34 shows the evolution of the wall pressure fluctuations for the slow and fast acoustic waves. The pressure variation for the slow mode shows that wall cooling reduced the growth of the first mode until $x=7$ inches and beyond this point the second mode grow exponentially. The disturbances generated by the fast mode remain almost constant up to $x=8$ inches and then grows exponentially. The maximum amplitudes reached are 0.0014 and 0.014 for the slow and fast acoustic waves, respectively. Hence, contrary to the adiabatic case the receptivity coefficient for the fast mode is 10 times larger than that for the slow wave. The amplitudes at the neutral point $x \sim 6.15$ inches, are $8.8^{*} 10^{-9}$ and $8.8^{*} 10^{-8}$ for the slow and fast acoustic waves, respectively. The receptivity coefficients are $4.4^{*} 10^{-3}$ and $4.4^{*} 10^{-2}$ for the slow and fast wave, respectively. Hence, the maximum receptivity coefficient is 100 times smaller for the fast wave compared to the adiabatic case. This is equivalent to an increase of 4.5 in the N-Factor at the transition onset in the cold wall case compared to the adiabatic wall case. Figure 35 shows the computed wavenumber variation along the streamwise direction for the slow and fast acoustic waves cases. The figure also includes the wavenumber distributions obtained from linear stability computations for the slow and the fast instability modes. The figure also shows the wavenumber variations for the slow and acoustic waves and for the vorticity waves. It is seen that the slow and fast acoustic waves generate the corresponding instability modes, slow and fast, near the leading edge of the cone. Further downstream between $x$ $\sim 4.5$ to 5.0 inches, the slow mode switches to the fast instability mode and the fast mode switches to vorticity waves. Close to $x \sim 6.8$ inches, the fast instability mode merges with the slow unstable mode. The vorticity wave switches to the slow unstable mode close to $x \sim 7.5$ inches. This shows that the generation of unstable second modes by the freestream disturbances is much different and more complex in the boundary layers over cold walls and over blunt cones as compared to a sharp cone with an adiabatic wall condition. As previously mentioned, modal analysis using adjoint methods can extract the different contents of the disturbances inside the boundary layer at a fixed location and could explain the observed different switching of modes from one to the other along the streamwise direction. 


\section{F. Sharp and Blunt Tip Flared Cones (Purdue)}

The model is a circular flared cone with a spherical nose region (Fig. 36). The concave circular flare has a radius of $3.0 \mathrm{~m}$. The nose radii are 0.16 and $1.0 \mathrm{~mm}$ for the sharp and blunt cones respectively. Computations were performed for a free-stream Mach number of 6 . The freestream stagnation temperature and pressure were $433 \mathrm{~K}$ and $12.2 \mathrm{kPa}(140 \mathrm{psi})$, yielding a unit Reynolds number of $10.3^{*} 10^{6} \mathrm{~m}^{-1}$. The simulations were also performed for a constant wall temperature of $300 \mathrm{~K}$. As with the previous model, this geometry was tested in the Boeing/AFOSR Mach 6 Quiet Tunnel at the same freestream conditions. ${ }^{13,14}$

\section{(1) Mean Flow and Linear Stability}

Detailed mean flow profiles and linear-stability results for the blunt flared cone were given in Ref. 58 . The mean surface pressure distributions along the axial direction for the straight cone as well as the sharp and blunt flared cones are depicted in Fig. 37(a). As expected, the pressure gradient is zero for the straight cone except for a region very close to the nose tip. The flare introduces an adverse pressure gradient along the surface, starting from the tip. There is a small difference in the pressure distribution near the nose region between the sharp and blunt flared cones. Beyond $x \sim 10 \mathrm{~cm}$, the pressure distributions are almost the same between the two cases. Figure 37(b) presents the boundary-layer density profiles at different axial locations for the sharp and blunt flared cones. Near the nose region, the boundary layers are thicker for the blunt cone case compared to that for the sharp cone case. Further downstream, the boundary-layer profiles are close to each other for both cases. The mean boundary-layer profiles showed that beyond an axial location of approximately $5 \mathrm{~cm}$, the boundary-layer thickness is observed to remain nearly constant at a thickness of $0.12 \mathrm{~cm}$ - a consequence of the balancing forces generated by the concave curvature of the flared-cone surface.

Figure 38(a) compares the growth rates for the most amplified waves for the four configurations, (1) straight sharp cone, (2) blunt circular flared cone, (3) sharp circular flared cone and (4) sharp flared cone. The growth rates for the sharp straight cone show the classical variation with a mildly stable first mode region near the nose region and a strongly unstable narrow second mode region downstream. The growth rates for the circular blunt flared cone show a strongly stable region up to $x=11.5 \mathrm{~cm}$ and a strongly unstable region downstream. The negative growth rates are about four times the maximum positive growth rates. The growth rates in the unstable region persist up to the end of the model with growth rates larger than that for the sharp straight cone. This is due to the slow variation of the boundary-layer profiles over the flare that is caused by the adverse pressure gradient. The growth rates for the sharp circular flared cone exhibit similar behavior to the sharp straight cone case up to $x=9 \mathrm{~cm}$ and farther downstream they exhibit similar behavior to the circular blunt flared cone case with growth rates slightly larger than that for the blunt case. Figure 38(b) shows the N-Factor curves for the most amplified waves for the blunt and sharp circular flared cones. For the blunt flared cone, the maximum N-Factor at the axial location $x \sim 400 \mathrm{~mm}$ is about 13.0, and the most amplified frequencies are about $279.0 \mathrm{kHz}$ (linear stability) and $286.0 \mathrm{kHz}$ (PSE). For the sharp flared cone, the maximum N-Factor is about 14.0, and the most amplified frequency is about $300 \mathrm{kHz}$. Transition was observed ${ }^{14}$ to occur for the sharp circular flared cone around $x=36 \mathrm{~cm}$. However, even with these large NFactors, transition was not observed on the blunt flared-cone model in the experiment. ${ }^{13}$

\section{(2) Interaction of Acoustic Waves with the flared cone}

As in the previous cases, after the mean flow was computed, two-dimensional acoustic disturbances (with an amplitude of $\tilde{p}_{a c} / p_{\infty}=1 * 10^{-6}$ ) were introduced at the outer boundary of the computational domain and timeaccurate simulations were performed. Simulations were performed for both slow and fast acoustic disturbances for a range of frequencies. Here, we discuss the results for a frequency of $292.5 \mathrm{kHz}$ for the blunt case and for a frequency of $300 \mathrm{kHz}$ for the sharp case. Figures 39(a-b) show the density fluctuations generated by the slow and fast acoustic waves for the blunt case and Fig. 39(c) depicts the results generated by the slow acoustic wave for the sharp case. The perturbation fields generated by the acoustic disturbances are qualitatively similar to that for the sharp-tipped straight cone.

Figures 40(a-c) show the evolution of the wall-pressure fluctuations on the flared cone that are induced by the slow and fast acoustic waves. The figures clearly show the generation and the eventual exponential growth of the instability waves inside the boundary layer. Figure 40(c) shows that the generation and the evolution of the disturbances generated by the slow acoustic wave for the sharp flared-cone are the same as that for the sharp-tipped straight cone (Fig. 19(a)). The wall pressure fluctuations for the sharp flared cone saturate at a value of $\left(p_{w} / p_{x}\right)=1.7$ around $x=36 \mathrm{~cm}$. This is equivalent to about $\left(p_{r m s} / p_{s}\right)=0.40$, where $p_{r m s}$ is the root mean square of the pressure fluctuations and $p_{s}$ is the mean surface pressure at $x=36 \mathrm{~cm}$. In the experiment, ${ }^{14}$ it was observed that the transition occurred on this model near $x \sim 36 \mathrm{~cm}$ and the maximum root-mean-square (RMS) value of the pressure was around 0.40 . 
Figure 40(a) shows the generation of disturbances by the slow acoustic wave for the blunt nose case. The important difference between the blunt flared cone and the sharp flared cone is the evolution of the first mode near the nose region. For the sharp flared cone, the first mode grows slightly in the downstream direction due to nonparallel effects in the boundary layer. However, for the blunt flared cone case, the disturbances decrease up to 70 $\mathrm{mm}$ and then remain constant up to $200 \mathrm{~mm}$ before smoothly transforming to the second mode, with subsequent exponential growth in the downstream direction. This behavior is linked to the entropy layer produced by the large bluntness of the flared cone geometry (Reynolds number of 10400 based on nose radius). Figures 41(a) and (b) show the density perturbations near the nose region for the blunt and sharp flared cone cases. It is clearly seen that the disturbances are confined closer to the wall region in the flow over the sharp flared cone compared to the blunt flared cone case. The results are similar to those observed in the flows over a blunt and a sharp straight cone Figs. 30 (a) and (b). Figure 40(b) shows the evolution of the wall pressure fluctuations on the blunt flared cone that are associated with the fast acoustic wave. In this case, the behavior is similar to that for the slow acoustic wave. However, the decay of the disturbances in the nose region is less than that for the slow acoustic wave. Therefore, the disturbances induced by the fast acoustic wave have higher amplitudes than those induced by a slow acoustic wave. In particular, the amplitude of the wall-pressure fluctuations at $x=40 \mathrm{~cm}$ are 0.0028 and 0.0055 for the slow and fast acoustic waves, respectively. The results of PSE calculations for the flared cone boundary layer are included in Figs. 40(a-c) for comparison to the computations. The computed disturbance growth, for both slow and fast acoustic waves, is observed to agree very well with the PSE results. As before, a receptivity coefficient was defined as the amplitude of the wall-pressure fluctuations at the neutral point non-dimensionalized by the freestream acoustic pressure, and the amplitude of the wall-pressure fluctuation at the neutral point was estimated by following the PSE results up to the neutral point. The receptivity coefficients for the slow and fast acoustic waves for the blunt flared cone, at a frequency of $292.5 \mathrm{kHz}$, at $x \sim 10 \mathrm{~cm}$ were found to be $4.9^{*} 10^{-3}$ and $9.3 * 10^{-3}$, respectively. The receptivity coefficient for the sharp flared cone at $x \sim 4 \mathrm{~cm}$ was found to be 1.2 . The receptivity coefficients for the blunt flared cone are about 130 times smaller than the receptivity coefficient for the sharp flared cone. This result agrees with our earlier receptivity calculations for sharp and blunt straight cones in hypersonic flows.

Figure 42 shows the computed wavenumber variation along the streamwise direction for the slow and fast acoustic waves cases for the blunt flared cone case. The results for the sharp flared cone are similar to the sharp straight cone case (Fig. 20) and are not discussed here. The figure also includes the wavenumber distributions obtained from linear stability computations for the slow and the fast instability modes as well as the wavenumber variations for the slow acoustic, fast acoustic, and vorticity waves based on the boundary-layer edge conditions. The first important observation is that neither the slow nor the fast acoustic waves generate any of the instability modes (neither the slow nor the fast mode), in the first part of the flare up to $x=20 \mathrm{~cm}$. The wavenumbers of the disturbances generated by the slow acoustic wave oscillate around the wavenumber for the vorticity waves. The wavenumbers of the disturbances generated by the fast wave closely follow the wavenumber for the fast acoustic wave. Close to $x=22 \mathrm{~cm}$, the wavenumber distribution for the disturbances generated by the slow acoustic wave jumps to the wavenumbers corresponding to the unstable second mode wavenumber obtained from the linearstability theory. Similarly, the wavenumber distribution for the disturbances generated by the fast acoustic wave jumps to the wavenumber distributions for the second mode close to $x=30 \mathrm{~cm}$. The important conclusion is that the unstable second mode is generated only in the downstream part of the flare. We do not know at this time the reasons or the mechanisms that caused these transformations to the unstable second mode at these specific locations and will explore them in the future.

Figure 43 presents a comparison between the computed and the measured amplitudes of the wall-pressure fluctuations at an axial location of $x=40 \mathrm{~cm}$. Here, the results for the computations correspond to fast acoustic waves over a range of frequencies. The measured wall-pressure spectrum was obtained in the experiment by Wheaton et al. ${ }^{14}$ As with the sharp-tipped cone, the frequencies and the shape of the computed amplitude spectrum compare favorably with the experimental data. In comparing amplitudes, however, the results are qualitative since the actual amplitudes of the freestream acoustic disturbances that were present in the experiment are unknown.

\section{G. Sharp Tipped Cone-Flare (NASA)}

This model consists of a $5^{\circ}$ half-angle sharp-cone region and a circular flared cone region (Fig. 44). The model is $50.8 \mathrm{~cm}$ (20 in.) long with a $25.4 \mathrm{~cm}$ (10 in.) front straight-cone region that is connected to a circular flared cone with a radius of $2.36 \mathrm{~m}$ (93.07 in.). At the juncture, the straight cone and the flared cone have the same slope. Computations were performed for a freestream Mach number of 6 . The freestream stagnation temperature and pressure were $400 \mathrm{~K}(810 \mathrm{R})$ and $11.33 \mathrm{kPa}(130 \mathrm{psi})$, yielding a unit Reynolds number of $8.950^{*} 10^{6} \mathrm{~m}^{-1}\left(2.727 * 10^{6}\right.$ $\left.\mathrm{ft}^{-1}\right)$. The simulations were performed for an adiabatic wall condition. This model geometry was tested in the NASA Langley Mach 6 Quiet Tunnel. ${ }^{9-11}$ 


\section{(1) Meanflow and stability}

Detailed results are given in Ref. (56). The mean surface pressure distribution along the axial direction of the cone is depicted in Fig. 37(a), and as expected, the adverse pressure gradient starts at the flared region of the model. The most amplified frequency for this model geometry, as determined by the linear-stability analysis, was found to be $220 \mathrm{kHz}$. The variation of the growth rate for this frequency is included in Fig. 38(a). The growth rates for the sharp flared cone exhibit similar behavior to the sharp cone case up to $x=16 \mathrm{~cm}$ and further downstream they exhibit similar behavior to the circular flared cone case. The maximum N-Factors obtained from the linear stability calculations and the PSE computations for the most amplified frequency of $220 \mathrm{kHz}$ are 7.5 and 11.5, respectively. This large difference between the N-Factors computed from the linear stability and the PSE occurs due to the growth of the disturbances in the nose region.

\section{(2) Interaction of Acoustic Waves with the Flared Cone}

With the mean flow computed, two-dimensional acoustic disturbances were introduced at the outer boundary of the computational domain and time-accurate simulations were performed. These simulations were performed for both slow and fast acoustic waves for a range of frequencies and we present the results for the slow acoustic wave. Here, we present the results for the most amplified frequency of $220 \mathrm{kHz}$. Figure 45 shows the evolution of the wall-pressure fluctuations on the flared cone that are induced by the slow acoustic wave. Here, data are shown for two freestream acoustic amplitude levels equal to $2 * 10^{-5}$ and $2 * 10^{-6}$. Similar to the sharp-tipped cone, the amplitude of the boundary-layer disturbances near the tip $(x \sim 0)$ is equal to the freestream acoustic amplitude. As expected, the disturbances are observed to grow from the tip region and then grow exponentially through the flared region of the cone. In comparison to the sharp-tipped cone case, the disturbance growth rate for the flared-cone model is significantly higher due to the adverse pressure gradient. In fact, the large growth rate leads to non-linear saturation at a pressure level of 1.8. It is interesting to note that the point at which saturation occurs increases by only $3 \mathrm{~cm}$ when the freestream acoustic amplitude is decreased by an order of magnitude. This small difference is a result of the large disturbance growth rate due to the adverse pressure gradient in the flared region of the cone. Experimental measurements on this flared cone model geometry (in the NASA Langley Mach 6 Quiet Tunnel) by Blanchard ${ }^{9}$ and Lachowicz et al. ${ }^{10}$ found transition to occur around $40 \mathrm{~cm}$. This is close to the axial location where non-linear saturation of the boundary-layer instabilities was observed in the present computations.

Figure 46 presents a comparison between the computed spectrum of maximum mass fluctuations $(\rho u)_{\max }$ and the measured disturbance spectrum in the boundary layer at an axial location of $x=40 \mathrm{~cm}$. Here, the results for the computations correspond to slow acoustic waves over a range of frequencies. The experimental data is an uncalibrated hot-wire voltage spectrum measured at the peak location in the boundary-layer disturbance profile. ${ }^{10}$ The peak frequency for the computed amplitude spectrum is $220 \mathrm{kHz}$, which compares well with the measured peak frequency of $226 \mathrm{kHz}$.

\section{Conclusions}

The receptivity of the instability waves to two-dimensional slow and fast acoustic and vortical waves at a free stream Mach number of 6 over a sharp-tipped wedge and a cone at adiabatic conditions, blunt cones at adiabatic conditions, sharp cones at cold wall conditions, sharp and blunt circular flared cones, and a sharp flared cone was numerically investigated. The effect of the incidence angles of the acoustic waves was also investigated. Both the steady and unsteady solutions were obtained by solving compressible Navier-Stokes equations using the $5^{\text {th }}$-order accurate weighted essentially non-oscillatory (WENO) scheme for space discretization and using a third-order totalvariation-diminishing (TVD) Runge-Kutta scheme for time integration. The major conclusions can be divided into four categories. (1) Flow over sharp geometries in adiabatic conditions, (2) Flow over blunt geometries, (3) Flow over sharp geometries with cold walls, and (4) Flow over flared geometries.

\section{- Flow over sharp wedges and cones in adiabatic wall conditions}

1. In hypersonic flows over sharp geometries with adiabatic wall conditions the slow and the fast acoustic waves directly synchronize with the slow and fast instability modes very close to the leading edge.

2. Forcing by slow acoustic waves is much more relevant in the transition process involved in supersonic and hypersonic boundary layers with adiabatic wall conditions.

3. For flows over wedges the receptivity coefficient for the slow mode based on the wall pressure fluctuations near the leading edge is about 2.5 times the amplitude of the freestream slow acoustic wave. 
4. The amplitude of the instability waves generated by the slow acoustic waves is about three times larger than that for the case of fast acoustic waves.

5. The receptivity on the leeward side increases slightly with increasing incidence angle and decreases on the windward side. The receptivity is the highest, by a factor of 1.8, for an incidence angle of 20 degrees.

6. The vortical disturbances also generate the second mode disturbances in hypersonic boundary layers. The receptivity coefficient is about four times smaller than that for the slow acoustic wave.

7. The wavenumber of the fluctuations showed that the vorticity waves generate the fast instability mode near the leading edge and the switching or the transformation of the fast instability mode to the slow instability mode in this case occurs near the continuous spectrum.

8. For flow over cones, the receptivity coefficient for the slow mode is about 5 times the amplitude of the freestream slow acoustic wave. It is also found that the amplitude of the instability waves generated by the slow acoustic waves is about eighty times larger than that for the case of fast acoustic waves.

9. The receptivity coefficient for the instability waves generated by the axi-symmetric vortical disturbances is about three times smaller than that for the slow acoustic wave.

\section{- Flow over blunt geometries}

1. In general, the bluntness stabilizes the linear instability of hypersonic boundary layers.

2. The stability characteristics strongly depend on the Reynolds number based on the nose radius and this is the critical parameter. For nose Reynolds numbers up to 10,000 , the stability characteristics are similar to the sharp nose cases and beyond that the stabilization of the boundary layer increases steeply with increasing nose Reynolds numbers.

3. The receptivity coefficients are orders of magnitude $\sim 10^{3}$ smaller than that for the sharp nose cases. This implies that the N-Factors at the transition onset in flows over blunt cones will be about 6 to 7 larger than that for the sharp cone. The reason for this is that in the blunt nose cases the freestream disturbances first enter the entropy layer and only further downstream enter the boundary layer.

\section{- Flow over sharp geometries with cold wall}

1. The wall cooling stabilizes the first mode strongly and destabilizes the second mode. The boundary layer becomes thinner and the frequencies of the most amplified waves increase.

2. Based on the local instability analysis, the transition onset in the cold wall cases move farther upstream compared to the adiabatic wall case.

3. Contrary to the adiabatic case, the receptivity coefficient for the fast mode is 10 times larger than that for the slow wave.

4. The receptivity coefficient is 100 times smaller for the fast wave compared to the adiabatic case. This is equivalent to an increase of 4.5 in the N-Factor at the transition onset in the cold wall case compared to the adiabatic wall case.

\section{- Flow over flared cones}

1. The adverse pressure gradients inhibit the growth of the boundary layer. This causes the unstable second mode region to persist for a long distance.

2. The frequency of the most amplified second-mode disturbance was shifted to a higher value relative to that for a straight cone, which has a zero pressure gradient.

3. The amplification of first- and second-mode disturbances is increased by the adverse pressure gradient.

4. The receptivity process for flow over the sharp flared cone is similar to that for the sharp straight cone.

5. Small bluntness decreases the receptivity coefficient by orders of magnitude $\sim 10^{2}$ compared to the sharp flared cone.

The next question is how one can incorporate these findings to improve the current N-Factor based transition prediction methods. To be successful, one has (1) to include the effects of freestream disturbances on the transition 
process in the transition prediction methods and, (2) to identify a criterion to determine the transition onset point. In this paper, the effects of freestream acoustic disturbances on the initial amplitudes of the stability waves were quantified. This is the first step in a difficult and complicated path to improving the existing transition prediction methods. If one can estimate the order of magnitude of the initial amplitudes, one may be able to determine the transition onset by answering the second question. The second question needs a concerted effort to come up with, if posssible, a criterion to determine the transition onset.

\section{Acknowledgments}

The author acknowledges his former students H. Zhao, S. Jeyasingham, and Kursat Kara for the contributions they made in these investigations. I also want to thank my colleques at NASA Langley Research Center Mike Kegerise, Lewis Owens, Rudy King, Frank Chen, George Beeler, Steve Wilkinson, Amanda Chou, Jenna Eppink, and Cathy McGinley from Flow Physics and Control Branch and Mujeeb Malik from Computational Aerosciences Branch for their contributions.

\section{References}

${ }^{1}$ Morkovin, M. V., Reshotko, E., and Herbert, T., "Transition in open flow systems - a reassessment," Bull. APS, Vol. 39, No. 9, pp. 1882, 1994.

${ }^{2}$ Lees, L., and Lin, C. C., "Investigation of the Laminar Boundary Layer in a Compressible Fluid," NACA Technical Note, Vol. 83, Sept. 1946, pp. 115.

${ }^{3}$ Lees, L., and Reshotko, E., "Stability of Compressible Boundary Layer," Journal of Fluid Mechanics, Vol. 12, 1962, pp. 455-477.

${ }^{4}$ Mack, L., "Boundary Layer Stability Theory,” JPL Report No. 900-277 Rev. A, Pasadena, Calif, Nov. 1969.

${ }^{5}$ Gaponov, M. C., and Weinstein, L. M., "The Influence of Flow Non-paralleism on Disturbance Development in the Supersonic Boundary Layer," Prc. Of the $8^{\text {th }}$ Canadian Congress of Applied Mechanics, 1981, pp. 673-674.

${ }^{6}$ Kendall,J. M., "Wind Tunnel Experiments Relating to Supersonic and Hypersonic Boundary-Layer Transition ," AIAA J., Vol. 13, No. 3, 1975, pp. 290-299.

${ }^{7}$ Stetson, K. F., Thompson, E. R., Donaldson, J. C., and Siler, L. G., "Laminar Boundary Layer Stability Experiments on a Cone at Mach 8, Part 1: Sharp Cone," AIAA 22 ${ }^{\text {nd }}$ Aerospace Sciences Meeting, Reno, Nevada, January 9-12, AIAA Paper 83$1761,1983$.

${ }^{8}$ Stetson, K. F., and Kimmel, R. L., “On Hypersonic Boundary-Layer Stability,” AIAA Paper 92-0737, January 1992.

${ }^{9}$ Blanchard, A.E., "An Investigation of Wall-Cooling Effects on Hypersonic Boundary-Layer Stability in a Quiet Wind Tunnel”, Ph.D. Dissertation, Old Dominion University, Dept. of Mechanical Engineering, Norfolk, VA, December, 1995.

${ }^{10}$ Lachowicz, J, T., Chokani, N., and Wilkinson, S.P., "Boundary-Layer Stability Measurements in a Hypersonic Quiet Tunnel”, AIAA Journal, Vol. 34, No. 12, December 1996.

${ }^{11}$ Wilkinson, S. P., "A Review of Hypersonic Boundary Layer Stability Experiments in a Quiet Mach 6 Wind Tunnel”, AIAA Paper 97-1819, June 1997.

${ }^{12}$ Maslov, A. A., Shiplyuk, A. N., Sidorenko, A. A and Arnal, D., "Leading Edge Receptivity of a Hypersonic Boundary layer on a Flat Plate," J. Fluid Mech., Vol. 426, 2001, pp. 73-94.

${ }^{13}$ Wheaton, B., Juliano, T.J., Berridge, D.C., Chou, A., Gilbert, P.L., Casper, K.M., Steen, L.E., Schneider, S., and Johnson, H.B., "Instability and Transition Measurements in the Mach 6 Quiet Tunnel," AIAA Paper 2009-3559, June 2009.

${ }^{14}$ Berridge, D.C., Chou, A., Ward, A. C., Steen, L.E., Gilbert, P. L., Juliano, T. J., Schneider, S., and Gronvall, J. E., "Hypersonic Boundary-Layer Transition Experiments in a Mach-6 Quiet Tunnel," AIAA Paper 2010-1061, January 2010.

${ }^{15}$ Casper, K.M., Beresh, S.J., Henfling, J.F., Spillers, R.W., Pruett, B., and Schneider, S.P., "Hypersonic Wind-Tunnel Measurements of Boundary-Layer Pressure Fluctuations," AIAA Paper 2009-4054, Jun 2009.

${ }^{16}$ Lees, L., "The Stability of the Laminar Boundary Layer in a Compressible Fluid," NACA Report 876, 1947.

${ }^{17}$ Demetriades, A., "New Experiments on Hypersonic Boundary Layer Stability Including Wall Temperature Effects," Proceedings of the Heat Transfer and Fluid Mechanics Institute, Vol. 26, 1978, pp. 39-54.

${ }^{18}$ Lysenko, V.I., Maslov, A.A. and Semenov, N.M., "Experimental Study of the Effect of Wall Heating on the Transition and Stability of Supersonic Boundary Layers," Fluid Mechanics - Soviet Research, Vol. 11 (6), 1982, pp. 32-43.

${ }^{19}$ Stetson, K.F., Thompson, E.R., Donaldson, J.C. and Siler, L.G., "Laminar Boundary Layer Stability Experiments on a Cone at Mach 8. Part 5 - Test with a Cooled Model," 20th Fluid Dynamics, Plasma Dynamics and Lasers Conference, Buffalo, NY, AIAA 1989-1895.

${ }^{20}$ Stetson, K. F., Thompson, E. R., Donaldson, J. C., and Siler, L. G., 1984, "Laminar Boundary Layer Stability Experiments on a Cone at Mach 8, Part 2: Blunt Cone," AIAA Paper, 0006.

${ }^{21}$ Reshotko, E. and Khan, M. M. S., September 1979, "Stability of the Laminar Boundary Layer on a Blunted Plate in Supersonic Flow", IUTAM Laminar-Turbulent Transition Symposium, Stuttgart, F.R.G.

${ }^{22}$ Malik, M. R., Spall, R. E., and Chang, C. L., 1990, "Effect of Nose Bluntness on Boundary Layer Stability and Transition," AIAA Paper, 0112.

${ }^{23} \mathrm{Hu}$, S. E., and Zhong, X., 1998, "Hypersonic Boundary-Layer Stability over Blunt Leading Edges with Bow-Shock Effects," AIAA Paper, 0433. 
$49^{\text {th }}$ AIAA Fluid Dynamics Conference, Aviation Forum , 2015, Dallas, Texas

\footnotetext{
${ }^{24}$ Zhnog, X., and Ma, Y., 2002, "Receptivity and Linear Stability of Stetson's Mach 8 Blunt Cone Stability Experiments," AIAA Paper, 2849.

${ }^{25}$ John, C. Evvard, Sept 5-13, 1956, "Transition studies on blunted bodies at supersonic speeds", Presented at 9th International Congress of Applied Mechanics, Brussels.

${ }^{26}$ Stetson, K. F., 1983, "Nosetip Bluntness Effects on Cone Frustum Boundary Layer Transition in Hypersonic Flow," AIAA
} Paper, 1763.

${ }^{27}$ Reshotko, E., 2006, “Transition Issues at Hypersonic Speeds,” AIAA Paper, 0707.

${ }^{28}$ Maslov, A. A., Mirinov, S. G., Shiplyuk, A. A., Sidorenko, A. A., Buntin, D. A., and Aniskin, V. M., 2002, "Hypersonic Flow Stability Experiments," AIAA Paper, 0153.

${ }^{29}$ Rufer, S. J., and Schneider, S. P., 2006, "Hot-Wire Measurements of Instability Waves on Cones at Mach 6," AIAA Paper, 3054 .

${ }^{30}$ Schneider, S. P., 2001, "Hypersonic Laminar Instability on Round Cones Near Zero Angle of Attack," AIAA Paper, 0206.

${ }^{31}$ Schneider, S. P., "Hypersonic Laminar-Turbulent Transition on Circular Cones and Scramjet Forebodies," Progress in Aerospace Sciences, Vol. 40, 2004, pp. 1, 50.

${ }^{32}$ Horvath, T. J., Berry, S. C., Hollis, B. R., Chang, C. C., and Singer, B. A., 2002, "Boundary Layer Transition On Slender Cones In Conventional And Low Disturbances Mach 6 Wind Tunnels," AIAA Paper, 2743

${ }^{33}$ Potter, J. L., 1974, Boundary Layer Transition on Supersonic Cones in an Aerobalistic Range, AIAA Journal, Vol. 13, No. $3,270-277$.

${ }^{34}$ Rosenboom, I., Hein, S., and Dallmann, U., 1999, "Influence of Nose Bluntness on Boundary Layer Instabilities in Hypersonic Cone Flows," AIAA Paper, 3591.

${ }^{35}$ Zhong, X., 2005, "Effect of Nose Bluntness on Hypersonic Boundary Layer Receptivity over a Blunt Cone," AIAA Paper, 5022 .

${ }^{36}$ Zhong, X. and Ma, Y., "Boundary-Layer Receptivity of Mach 7.99 Flow over a Blunt Cone to Free-Stream Acoustic Waves,” J. Fluid Mech. , Vol. 556, 2006, pp. 55, 103, doi:10.1017/S0022112006009293.

${ }^{37}$ Balakumar, P., 2006, "Stability of Supersonic Boundary Layers over Blunt Wedges," AIAA Paper, 3053.

${ }^{38}$ Balakumar, P. and Malik, M.R., "Effects of Adverse Pressure Gradient and Wall Cooling Instability of Hypersonic Boundary Layers," HTC-9404, 1994.

${ }^{39}$ Goldstein, M. E., "The evolution of Tollmien-Schlichting waves near a leading-edge”, J. Fluid Mechanics, Vol. 127, 1983, pp. 59-81.

${ }^{40}$ Goldstein, M. E., "Scattering of acoustic waves into Tollmien-Schlichting wavesby small streamwise variations in surface geometry", J. Fluid Mechanics, Vol. 154, 1985, pp. 509-529.

${ }^{41}$ McKenzie, J. F., and Westphal, K. O., "Interaction of Linear Waves with Oblique Shock Waves," Physics of Fluids, Vol. 11, No. 11, 1968, pp. 2350-2362.

${ }^{42}$ Mack, L. M., "On the application of linear stability theory and the problem of supersonic boundary-layer transition," $A I A A$ Journal., 13, No. 3, 1975.

${ }^{43}$ Gaponov, S. A., "Interaction between a supersonic boundary layer and acoustic disturbances," Fluid Dynamics, 6, 1977.

${ }^{44}$ Gaponov, S. A. and Smorodsky, B. V., "Supersonic Boundary Layer Receptivity to Streamwise Acoustic Field," IUTAM Symposium, Spinger-Verlag, 1999.

${ }^{45}$ Gushin, V. R., and Fedorov, A. V., "Excitation and development of unstable disturbances in supersonic boundary layer," Fluid Dynamics, Vol. 25, No. 3, 1990, pp. 344-352.

${ }^{46}$ Fedorov, A. V., and Khokhlov, A. P., "Prehistory of Instability in a Hypersonic Boundary Layer," Theoret. Comput. Fluid Dynamics, Vol. 14, pp. 359-375, 2001.

${ }^{47}$ Fedorov, A. V., and Khokhlov, A. P., “ Excitation of Unstable Modes in a Supersonic Boundary Layer by Acoustic Waves,” Fluid Dynamics, Vol. 26, No. 4, pp. 531-537, July 1991.

${ }^{48}$ Fedorov, A. V., and Khokhlov, A. P., "Sensitivity of a Supersonic Boundary Layer to Acoustic Disturbances," Fluid Dynamics, No. 27, pp. 29-34, 1992

${ }^{49}$ Fedorov, A.V., "Receptivity of a High-Speed Boundary Layer to Acoustic Disturbances," Journal of Fluid Mechanics, Vol. 491, 2003, pp. 101-129.

${ }^{50} \mathrm{Ma}$, Y. and Zhong, X., "Receptivity of a Supersonic Boundary Layer over a Flat Plate. Part 1. Wave Structures and Interactions," Journal of Fluid Mechanics, Vol. 488, 2003, pp. 31-78.

${ }^{51}$ Ma, Y. and Zhong, X., "Receptivity of a Supersonic Boundary Layer over a Flat Plate. Part 2. Receptivity to Freestream Sound," Journal of Fluid Mechanics, Vol. 488, 2003, pp. 79-121.

${ }^{52}$ Egorov, I.V., Fedorov, A.V. and Soudakov, V.G., "Receptivity of a Hypersonic Boundary Layer over a Flat Plate with a Porous Coating," Journal of Fluid Mechanics, Vol. 601, 2008, pp. 165-187.

${ }^{53}$ M M. R. Malik and P. Balakumar, “Acoustic receptivity of Mach 4.5 boundary layer with leading edge bluntness," Theo. Comput. Fluid Dyn., September 2007, Vol 21, Number 5, pp:323-342.

${ }^{54}$ Kara, K., Balakumar, P., and Kandil, O.A., "Receptivity of Hypersonic Boundary Layers Due to Acoustic Disturbances over Blunt Cone," AIAA Paper 2007-945, Jan. 2007.

${ }^{55}$ Kara, K., Balakumar, P., and Kandil, O.A., "Effects of Nose Bluntness on Stability of Hypersonic Boundary Layers over a Blunt Cone," AIAA Paper 2007-4492, June 2007.

${ }^{56}$ Kara, K., Balakumar, P., and Kandil, O.A., "Effects of Wall Cooling on Hypersonic Boundary Layer Receptivity over a Cone," AIAA Paper 2008-3734, June 2008. 
${ }^{57}$ Kara, K., Balakumar, P. and Kandil, O. A., "Effects of Nose Bluntness on Hypersonic Boundary Layer Receptivity and Stability over Cones," AIAA Journal, Vol. 49, No. 12, December 2011, pp. 2593-2606.

${ }^{58}$ Balakumar, P., and Kegerise, M. A., "Receptivity of Hypersonic Boundary Layers over Straight and Flared Cones," AIAA Paper 2010-1545, January 2010.

${ }^{59}$ P. Balakumar, and Owens, L. R., "Stability of Hypersonic Boundary Layers on a Cone at an Angle of Attack," AIAA 2010-4718, 40 ${ }^{\text {th }}$ AIAA Fluid Dynamics Conference, Chicago, Illinois, June 28- July 1, 2010.

${ }^{60}$ P. Balakumar, and Kegerise, M. K., "Receptivity of Hypersonic Boundary Layers to Acoustic and Vortical Disturbances," AIAA 2011-0371, 49 $9^{\text {th }}$ AIAA Aerosciences Meeting, Orlando, Florida, January 4-7, 2011.

${ }^{61}$ P. Balakumar, "Receptivity of Hypersonic Boundary Layers to Distributed Roughness and Acoustic Disturbances", $50^{\text {th }}$ AIAA Aerosciences Meeting, Dallas, TX, January 7-10, 2013.

${ }^{62} \mathrm{Shu}$, C.W., "Essentially Non-Oscillatory And Weighted Essentially Non-Oscillatory Schemes For Hyperbolic Conservation Laws", NASA/CR-97-206253 and ICASE Report No. 97-6.

${ }^{63}$ Atkins, H.L., "High-Order ENO Methods for the Unsteady Compressible Navier-Stokes Equations," AIAA Paper 91-1557, 1991.

${ }^{64}$ Balakumar, P., Zhao, H., and Atkins, H., "Stability of Hypersonic Boundary Layers Over a Compression Corner," AIAA Paper 2002-2848, June 2002. 


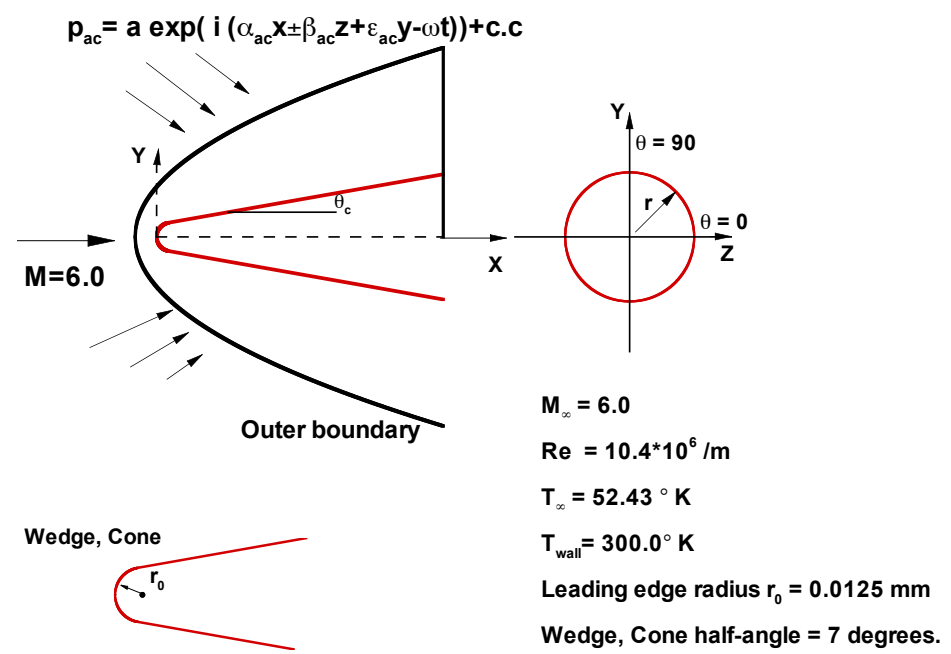

Figure 1. Schematic diagram of the computational model.



Figure 2. Mean density, velocity profiles and the generalized velocity derivative obtained from the similarity solution. 
(a)

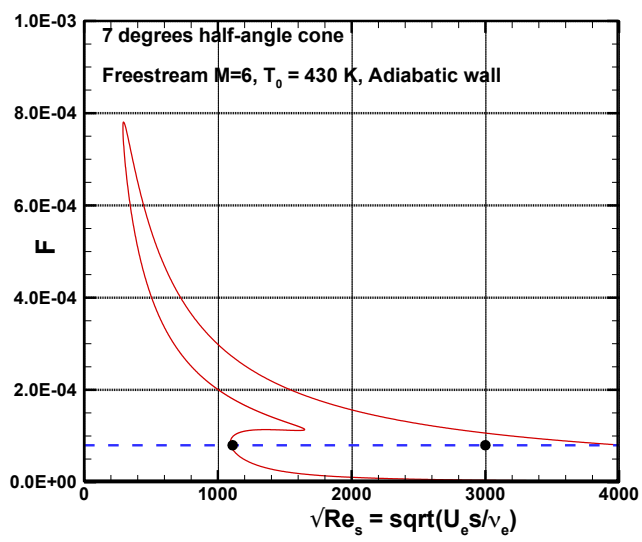

(b)

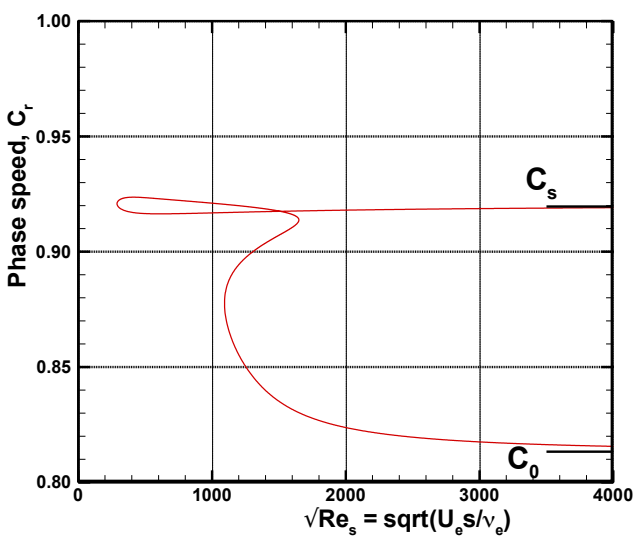

Figure 3. Neutral stability diagram for the similarity profiles. (a) $\left(\sqrt{ } \mathrm{Re}_{\mathrm{s}}, \mathrm{F}\right)$ plane, and (b) $\left(\sqrt{ } \mathrm{Re}_{\mathrm{s}}, \mathrm{C}_{\mathrm{r}}\right)$ plane.

(a)

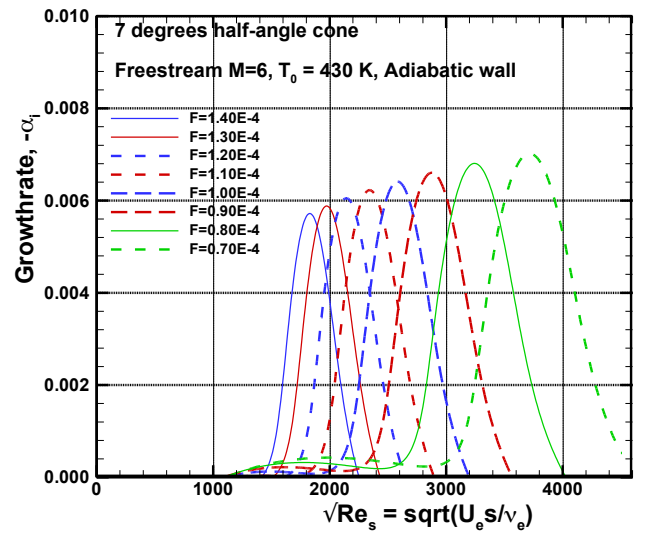

(b)



Figure 4. (a) Growth rate and (b) N-Factor curves computed from the linear stability analysis.

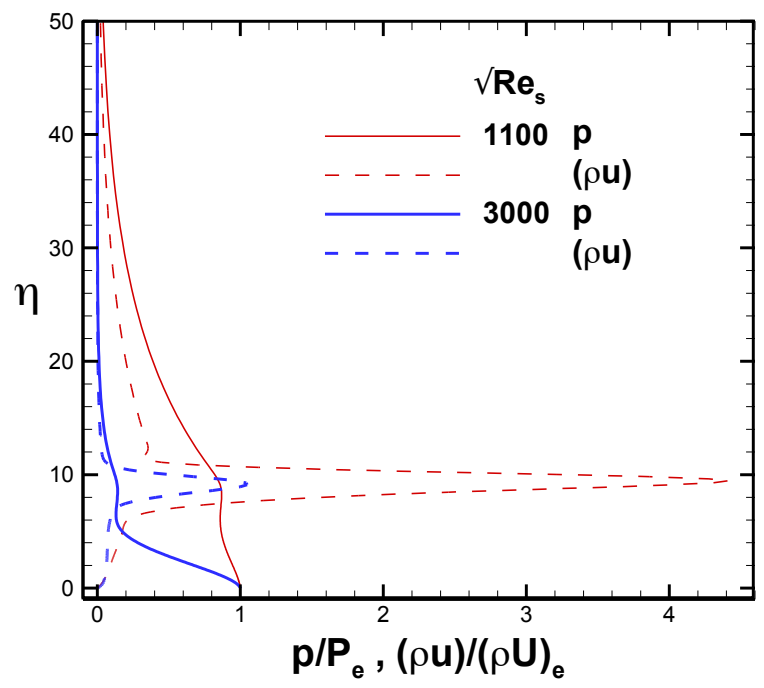

Figure 5. Eigenfunction distributions for the pressure and mass-flux perturbations at two station $\sqrt{ } \operatorname{Re}_{\mathrm{s}}=1100$ and 3000 . $F=0.8 E-4$. 
(a)

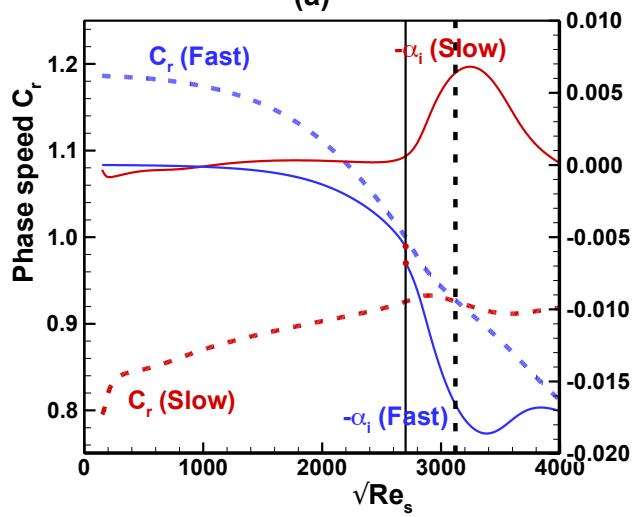

(b)

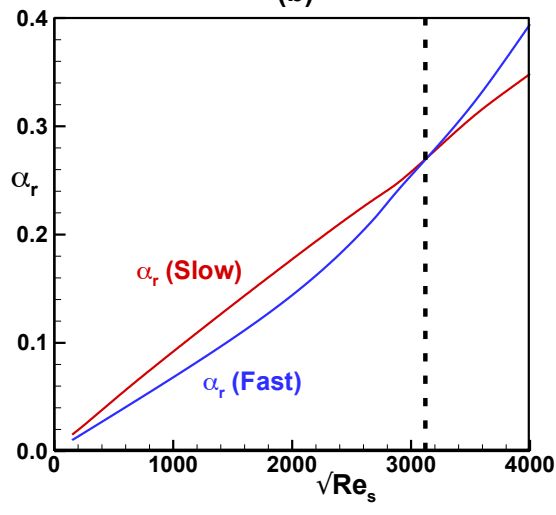

Figure 6. Eigenvalues from the linear stability computations for fast (blue) and slow (red) modes for the frequency $F=0.80 * 10^{-4}$. (a) Phase speed $c_{r}$ and growth rates $-\alpha_{i}$ and (b) wavenumber $\alpha_{r}$ and wavelength.

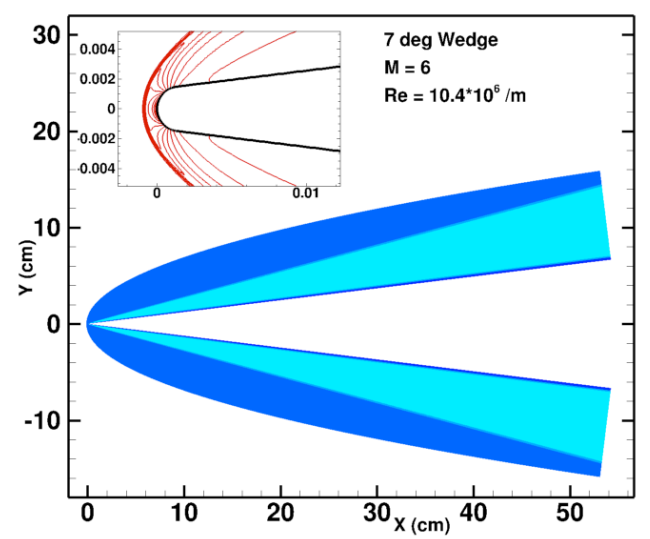

Figure 7. Mean density contours for flow over a 7-degree sharp-tipped wedge at Mach 6.

(a)

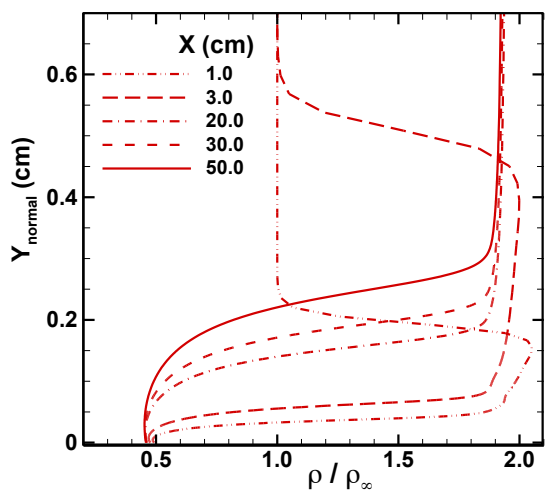

(b)

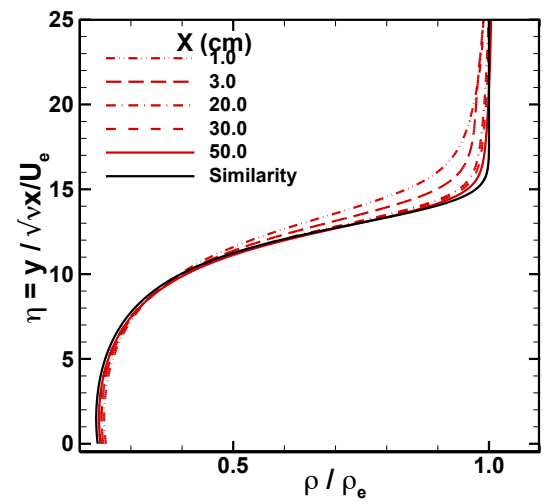

Figure 8. Boundary layer density profiles (a) in physical and (b) similarity coordinates at different axial locations for flow over a 7-degree sharp-tipped wedge at Mach 6. 

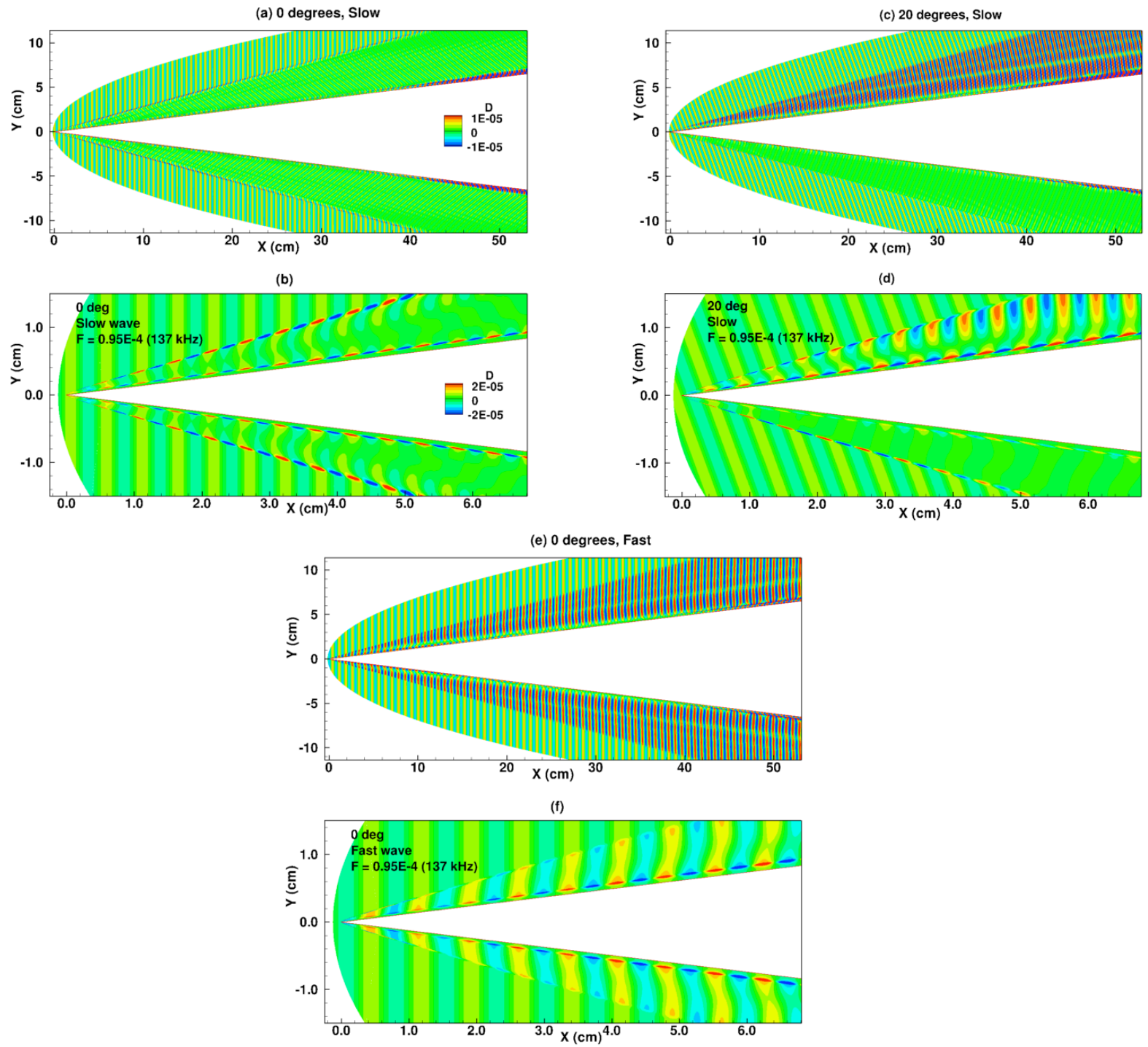

Figure 9. Density fluctuations generated by the interaction of a two-dimensional acoustic wave with the sharp-tipped wedge, incident angles 0 and 20 degrees. $F=0.95 * 10^{-4}$. 

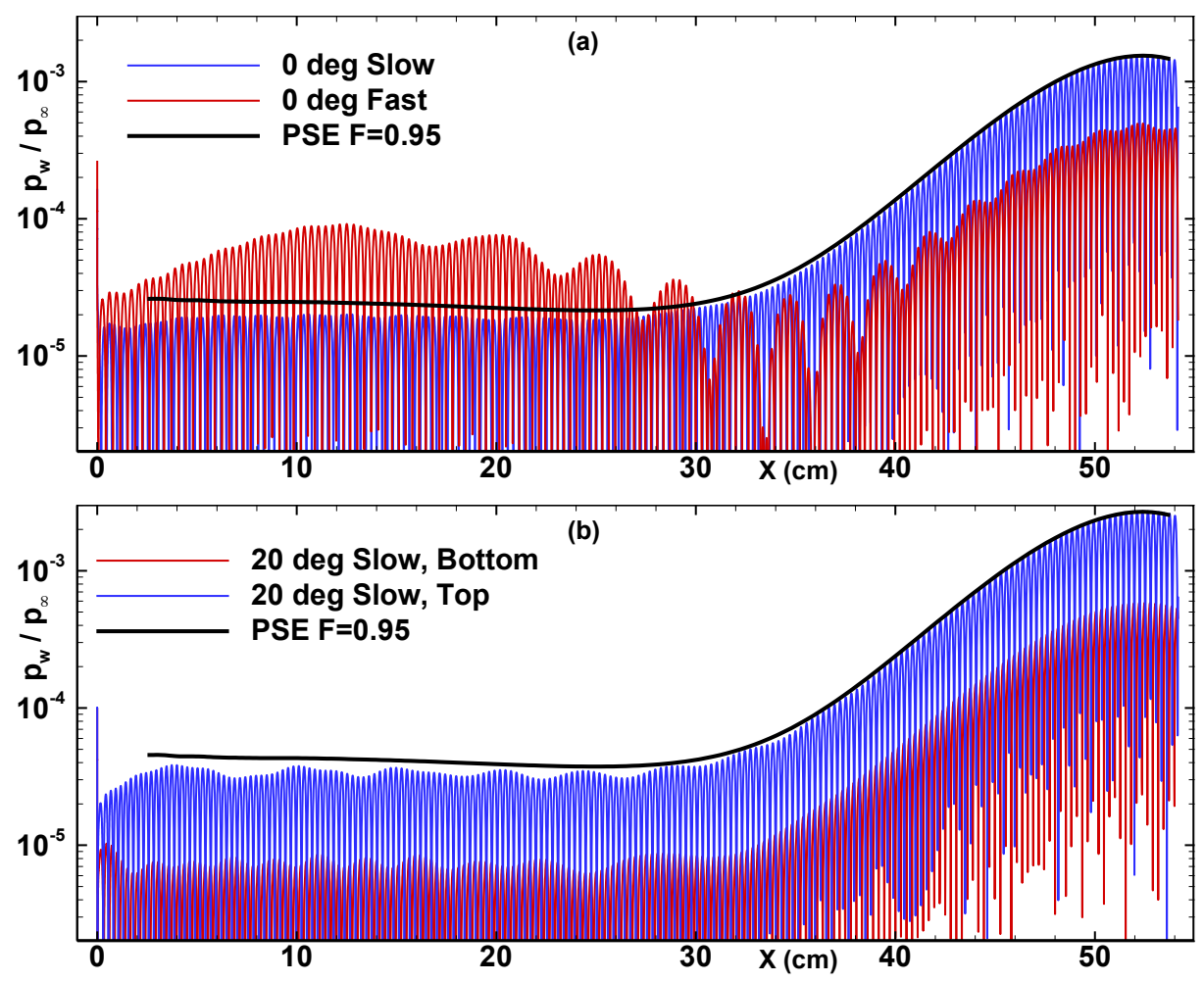

Figure 10. Pressure fluctuations at the top and bottom surfaces of the sharp-tipped wedge generated by twodimensional slow and fast acoustic waves at incident angles of 0 and 20 degrees. $F=0.95 * 10^{-4}$.
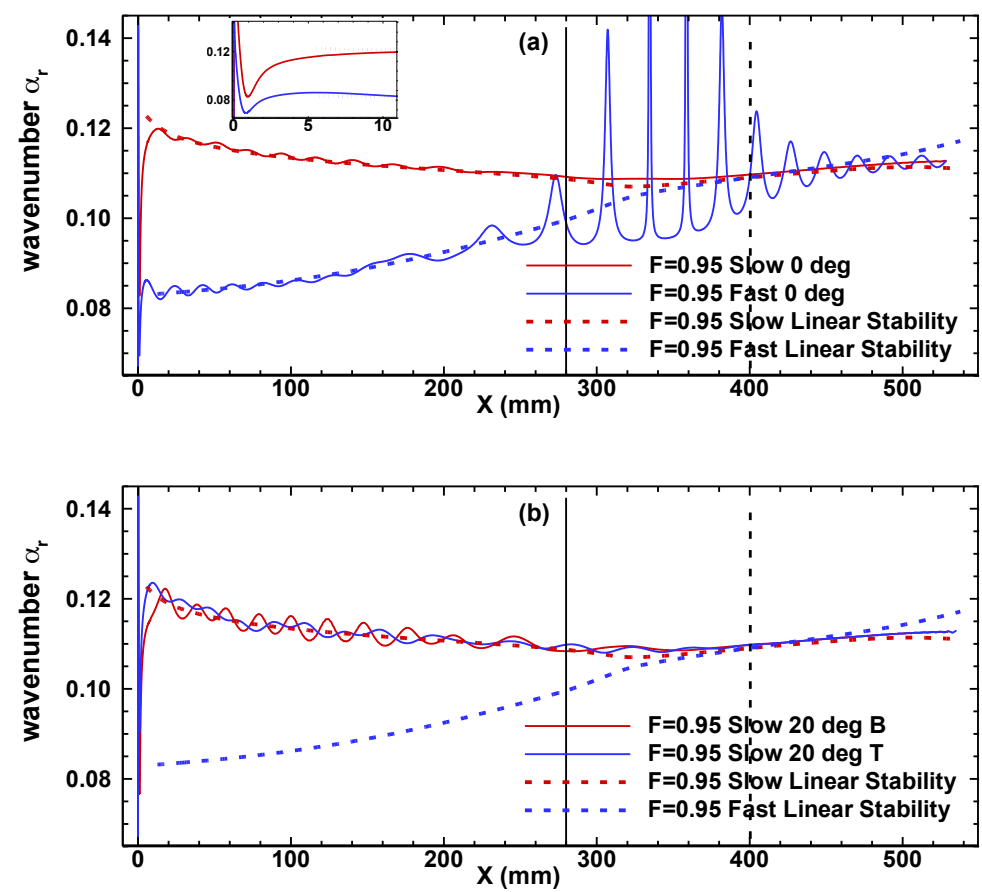

Figure 11. Wavenumber of the pressure fluctuations along the wall generated by two-dimensional acoustic waves at incident angles of 0 and 20 degrees and comparison with the linear stability. $F=0.95 * 10^{-4}$. Solid line is the location of the continuous spectrum; dotted line is the synchronization point. 

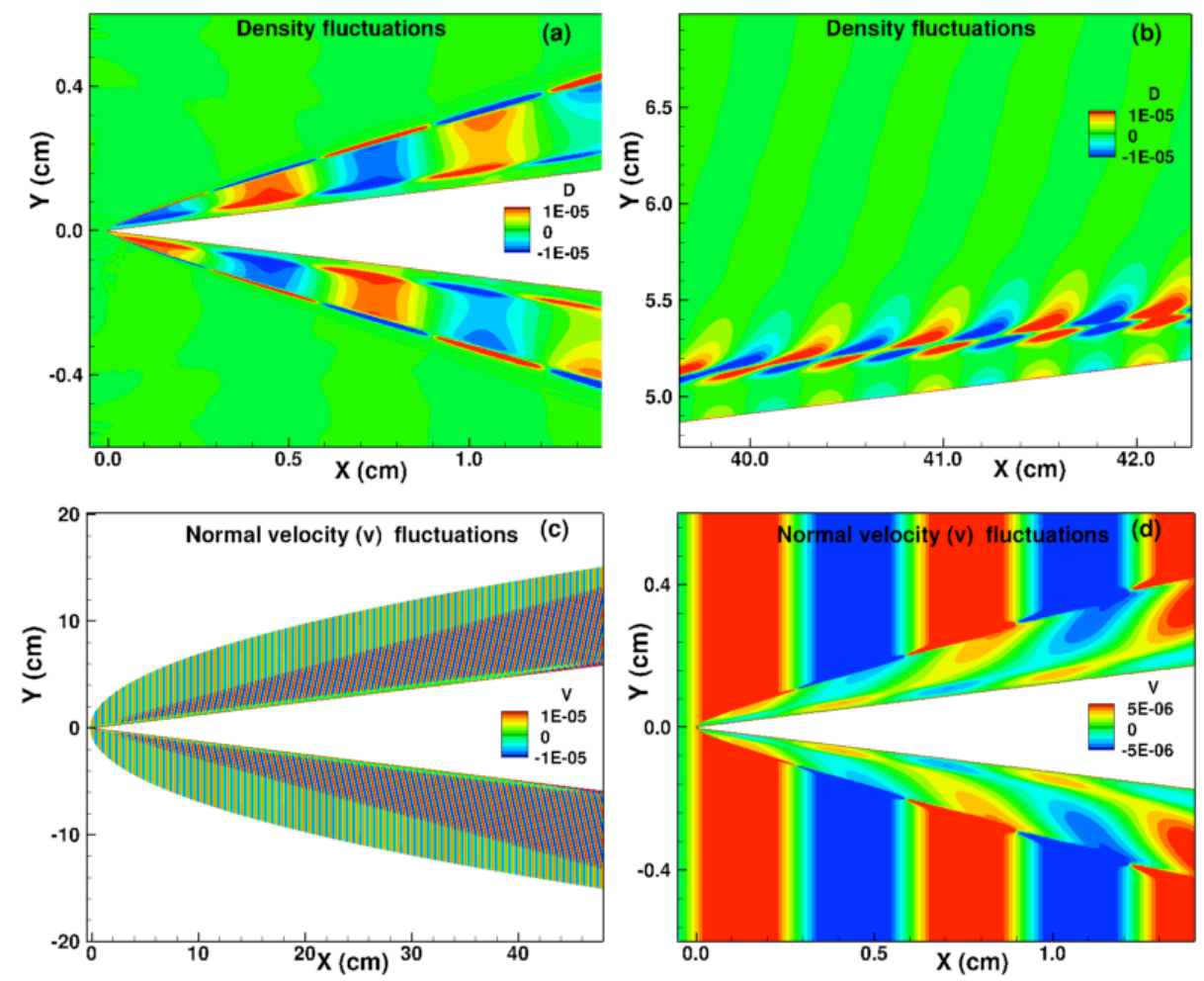

Figure 12. Density and vertical velocity, $v$, fluctuations generated by the interaction of a two-dimensional vortical disturbance with the sharp-tipped wedge. $F=0.95 * 10^{-4}$.

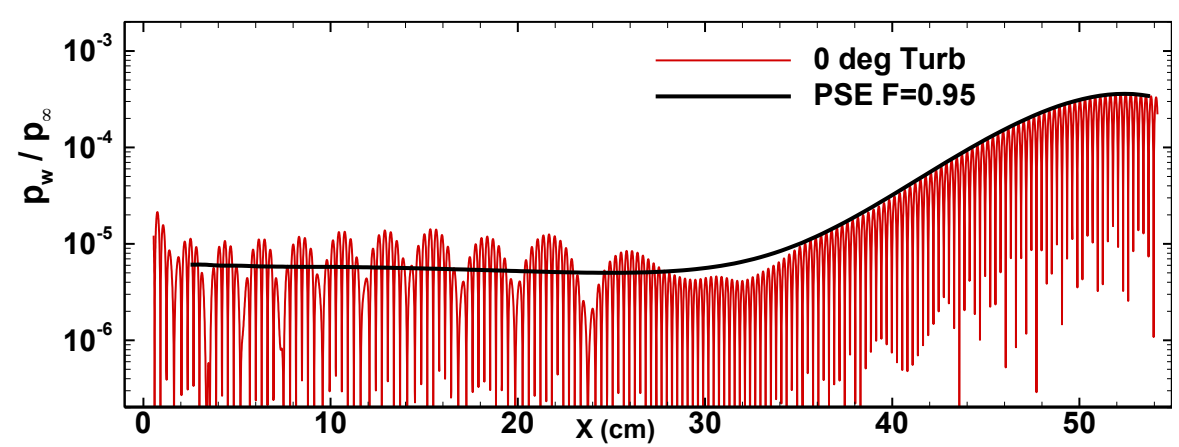

Figure 13. Wall pressure fluctuations generated by the interaction of two-dimensional vortical disturbances with the wedge. $F=0.95 * 10^{-4}$. 


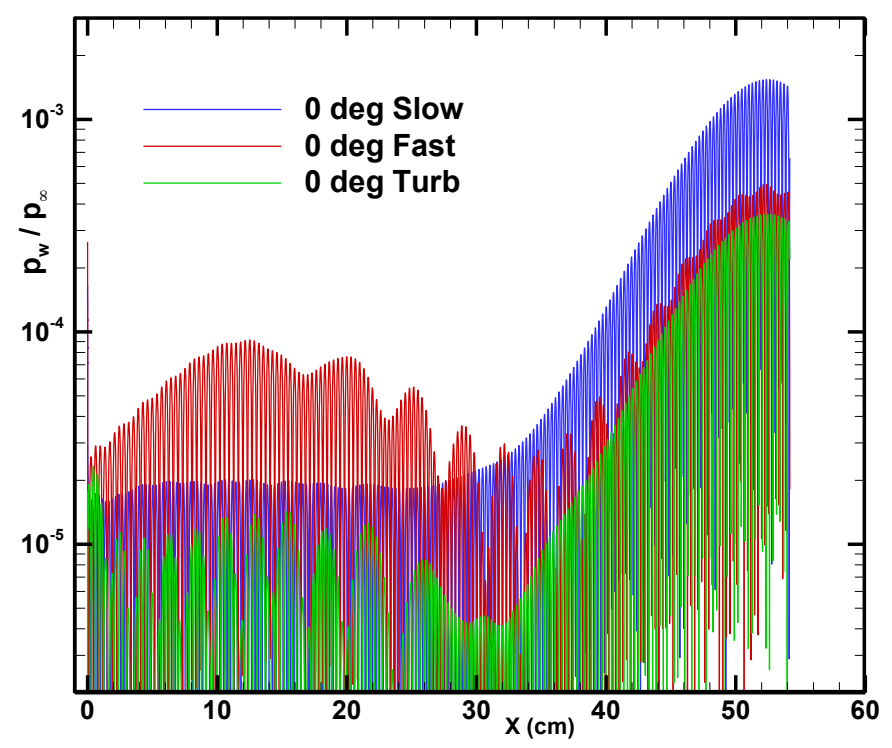

Figure 14. Comparison of the wall-pressure fluctuations along the surface of the wedge generated by the slow and fast acoustic waves, and by the vortical disturbances. $F=0.95 * 10^{-4}$.

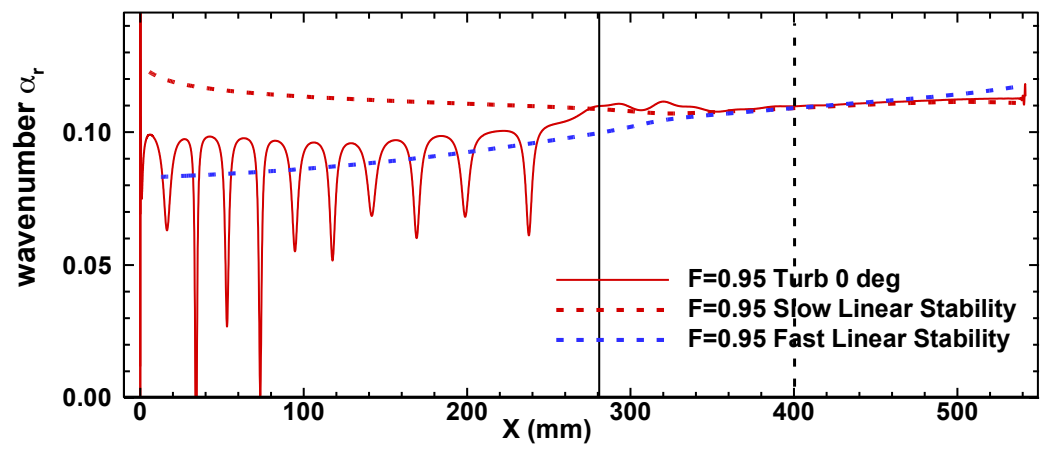

Figure 15. Wavenumber of the pressure fluctuations along the wall generated by the interaction of twodimensional vortical disturbances with the wedge and comparison with the linear stability. $F=0.95 * 10^{-4}$
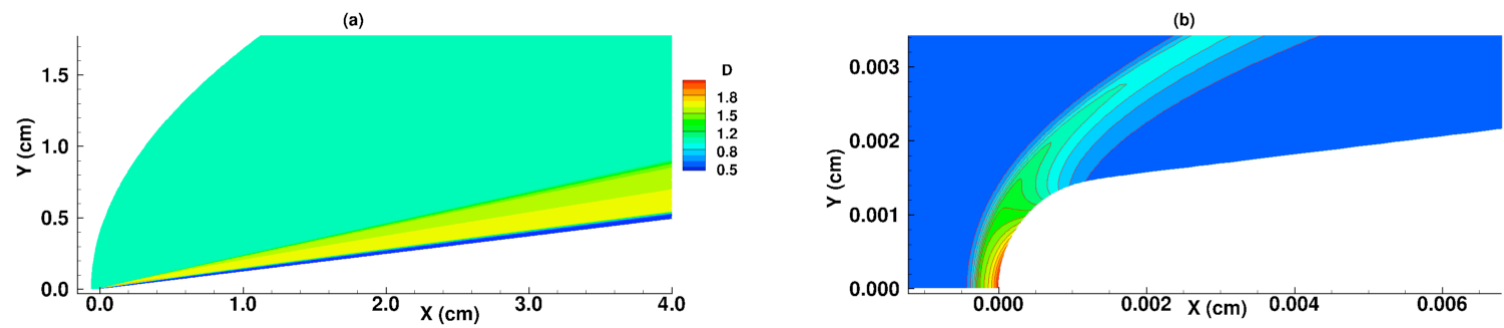

Figure 16. Mean density contours for flow over a 7-degree sharp-tipped cone at Mach 6. (a) Nose and the cone regions and (b) nose region. 




Figure 17. N-Factor curves computed from the linear stability analysis and the PSE analysis based on ( $\rho$ u $)_{\text {max }}$.



(b)

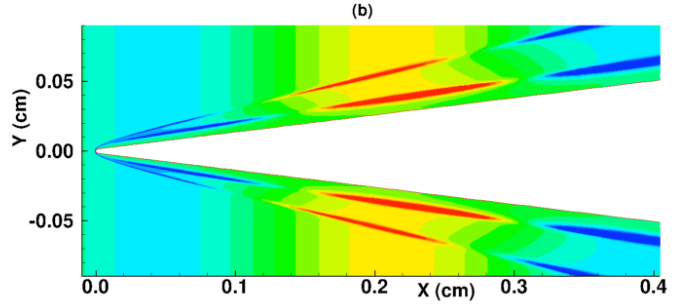

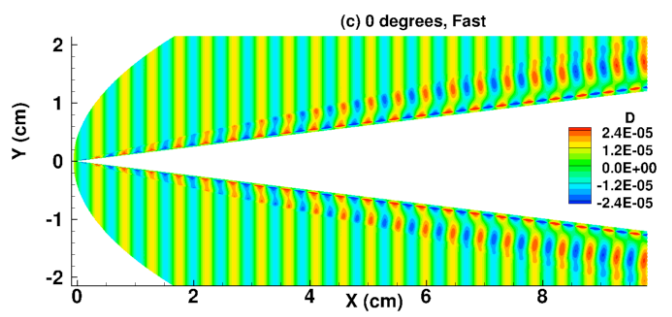

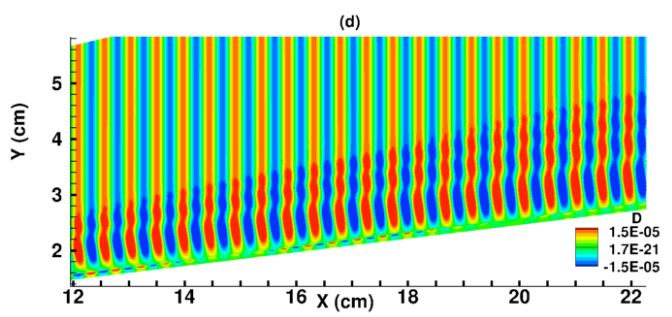

Figure 18. Density fluctuations generated by the interaction of a two-dimensional plane slow (a, b) and fast (c, d) acoustic waves with the sharp-tipped cone, incident angle 0 degrees. $F=1.5 * 10^{-4}$. 



Figure 19. Wall-pressure fluctuations along the sharp-tipped cone obtained from the DNS for twodimensional plane (a) slow and (b) fast acoustic waves.

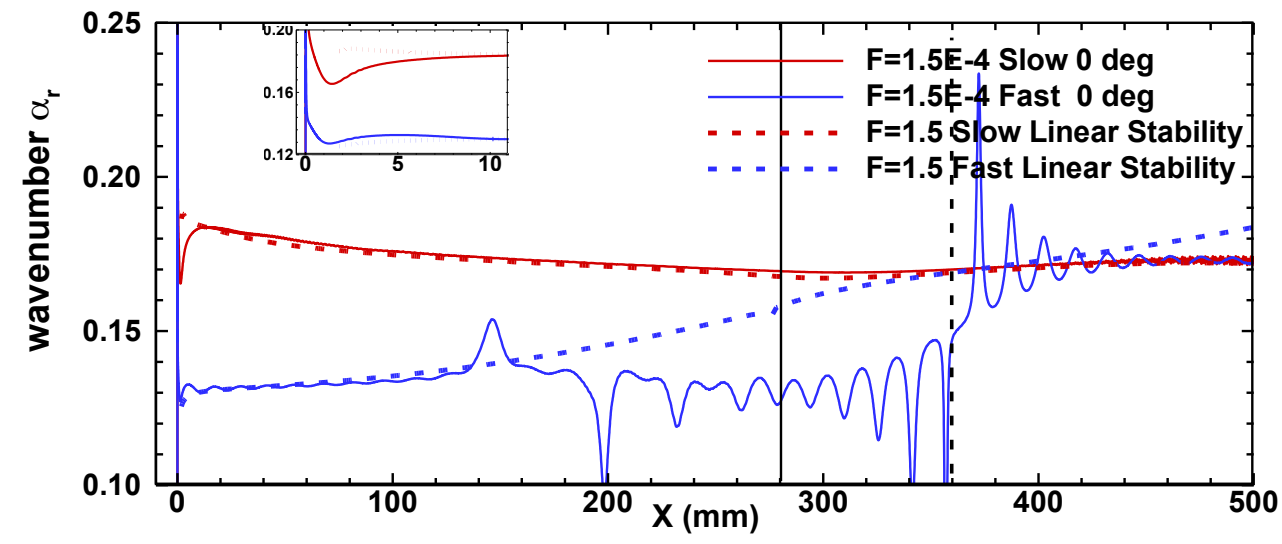

Figure 20. Wavenumber of the pressure fluctuations along the wall and comparison with the linear stability. $F=1.5 * 10^{-4}$. 
(a) $X=208 \mathrm{~mm}$ (Noisy)

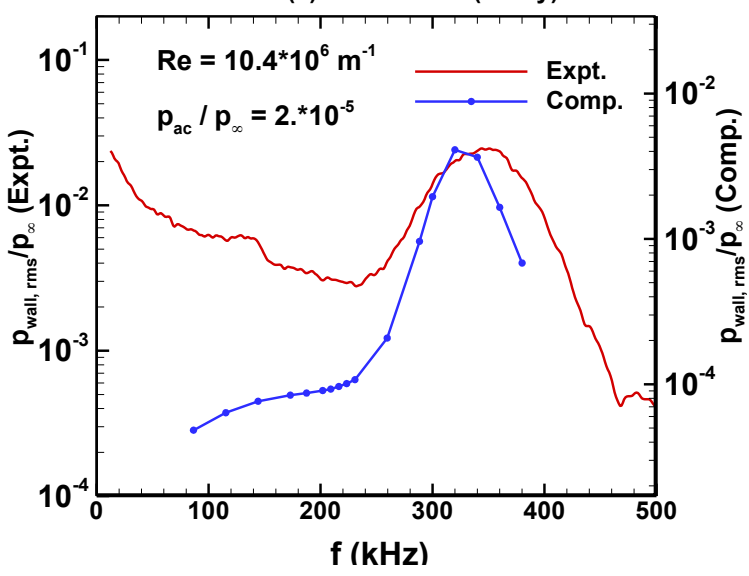

(b) $X=\mathbf{4 9 0} \mathrm{mm}$ (Quiet)

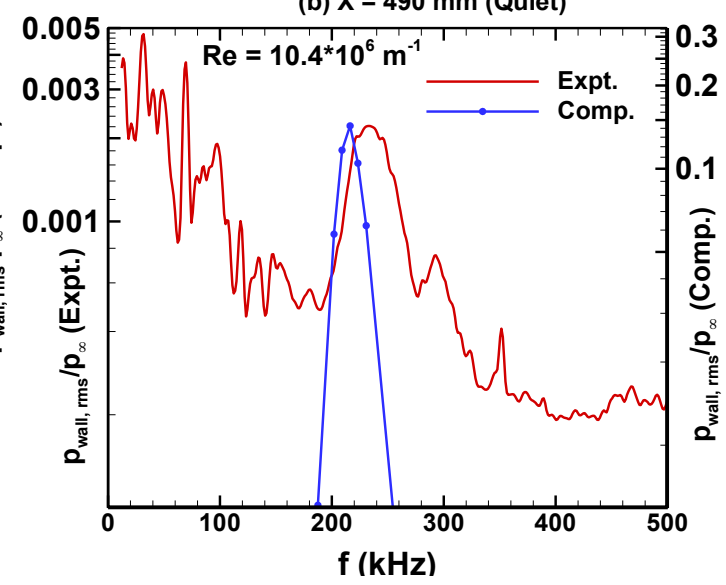

Figure 21. Comparison between the computed and measured wall-pressure spectrum for the sharp-tipped cone at a) $x=208 \mathrm{~mm}$ and b) $x=490 \mathrm{~mm}$. Experimental data from Casper et. al. ${ }^{15}$.

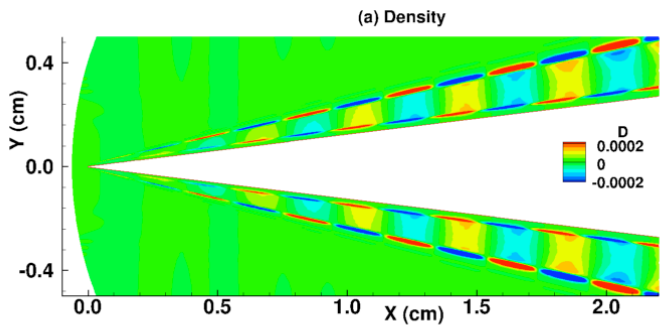

(b)



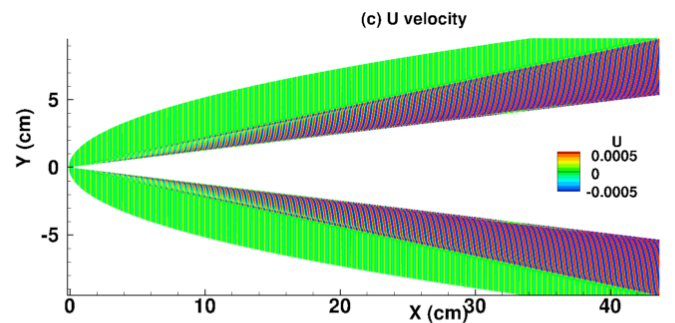

(d)

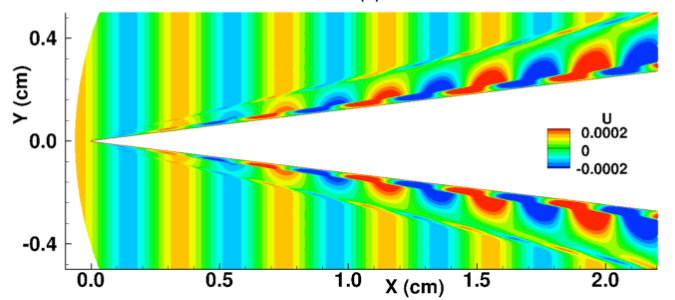

Figure 22. Density (a, b) and u velocity $(c, d)$ fluctuations generated by the interaction of an axi-symmetric vortical disturbance with the sharp-tipped cone. $F=1.5 * 10^{-4}$

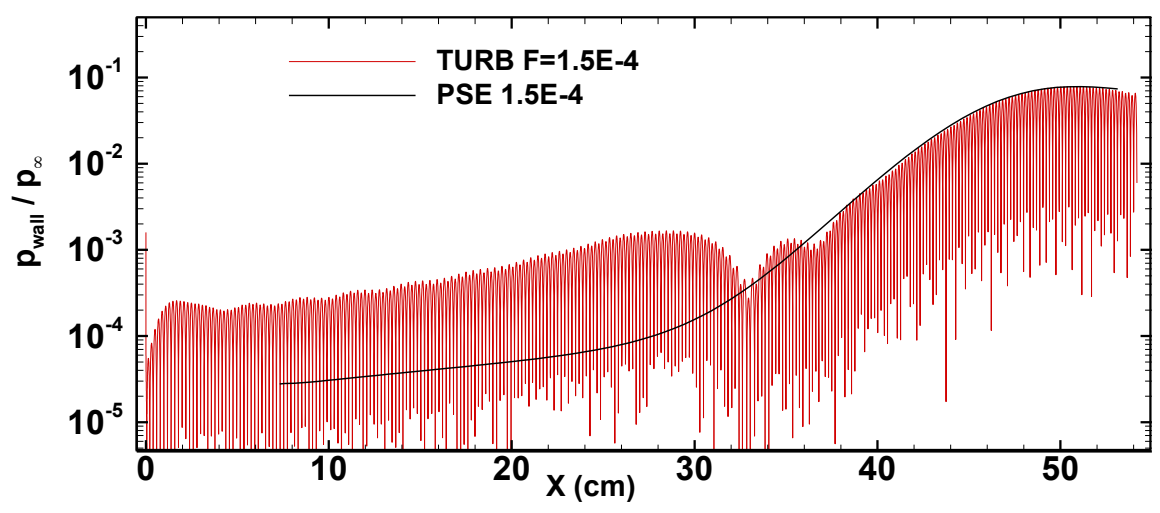

Figure 23. Wall pressure fluctuations generated by the interaction of an axi-symmetric vortical disturbances with the cone. $F=1.5 * 10^{-4}$. 


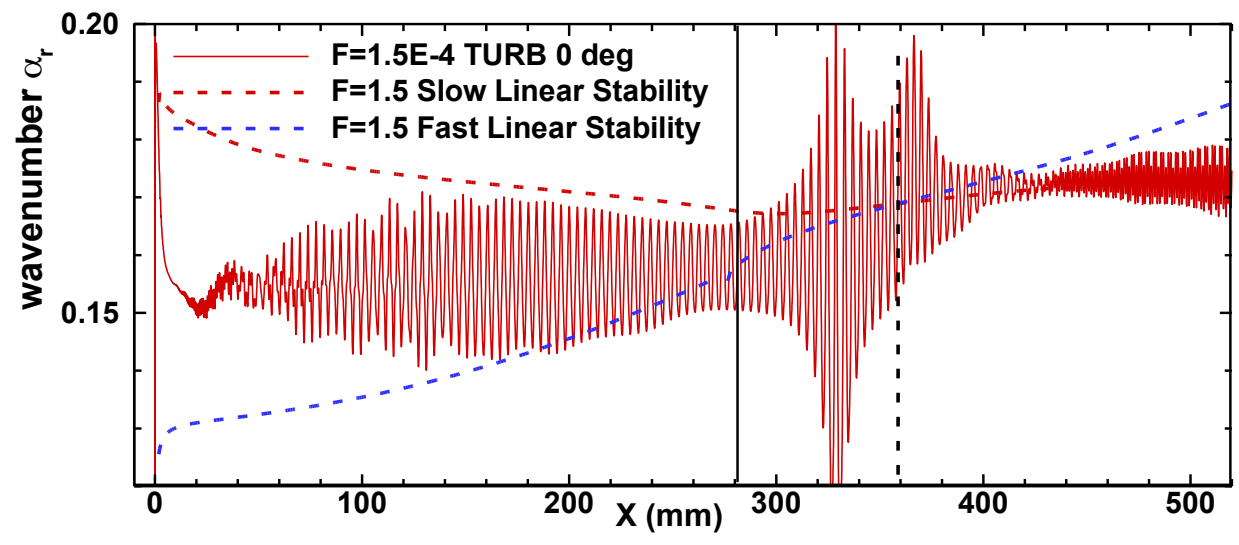

Figure 24. Wavenumber of the pressure fluctuations along the wall generated by the interaction of an axisymmetric vortical disturbance with the sharp-tipped cone and comparison with the linear stability. $F=1.5 * 10^{-4}$
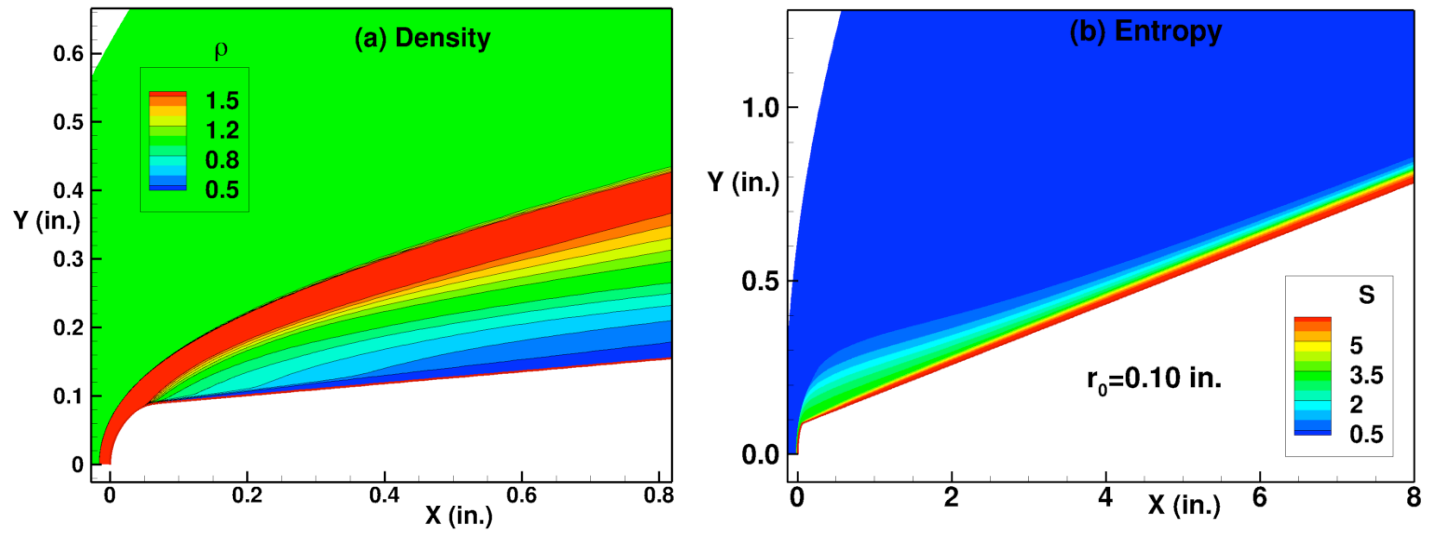

Figure 25. Contours of (a) density and (b) entropy (not to scale) for flow over a blunt cone with a nose radius of $r_{0}=0.10$ in. $R e=7.8 \times 10^{6} / \mathrm{ft}$.

(a) $r_{0}=0.001$ in.



(b) $r_{0}=0.10$ in.

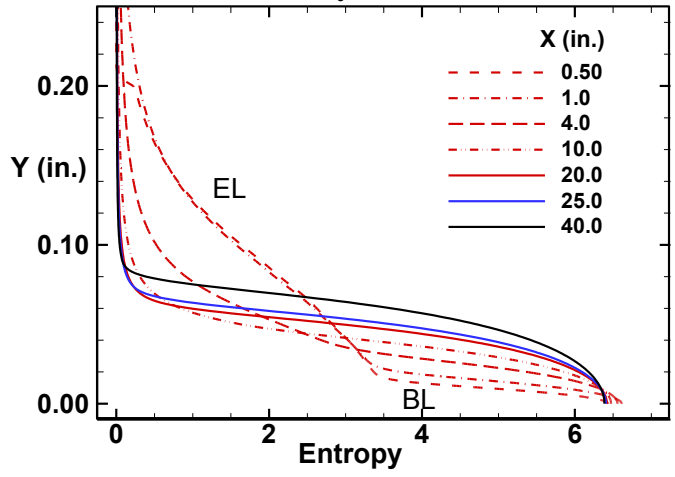

Figure 26. Entropy profiles at different $X$ stations for (a) $r_{0}=0.001$ in. and (b) $r_{0}=0.10$ in. $\operatorname{Re}=7.8 \times 10^{6} / f t$. 
(a)

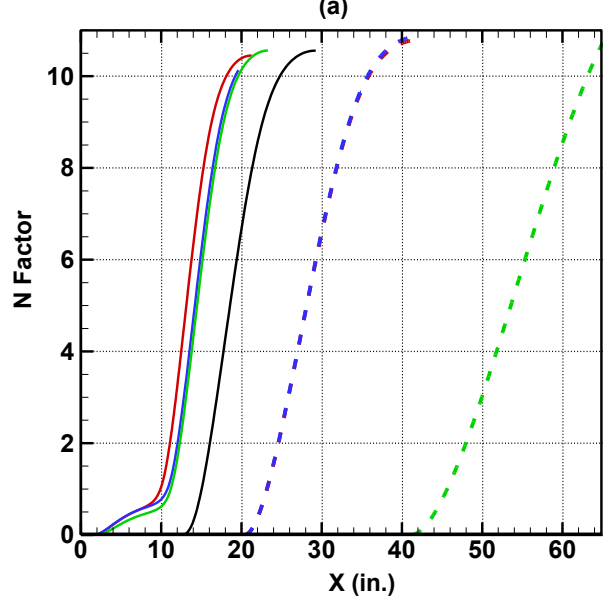

(b)

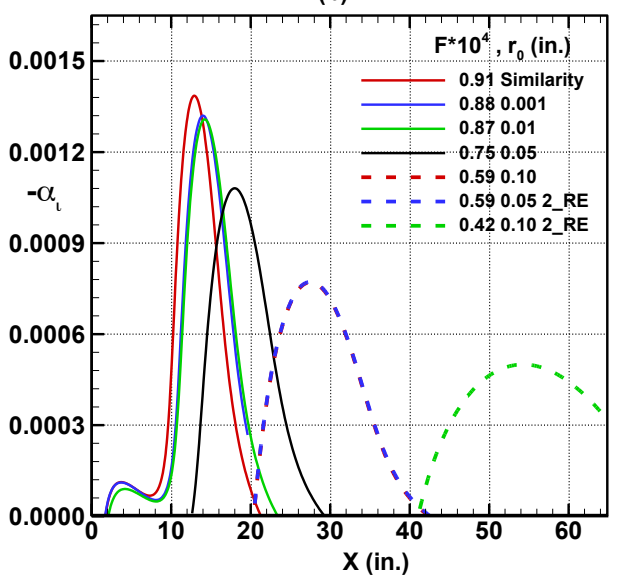

Figure 27. Computed (a) N-Factors and (b) growth rates for the most amplified disturbances for $r_{0}=0.001,0.01,0.05,0.10$ in.

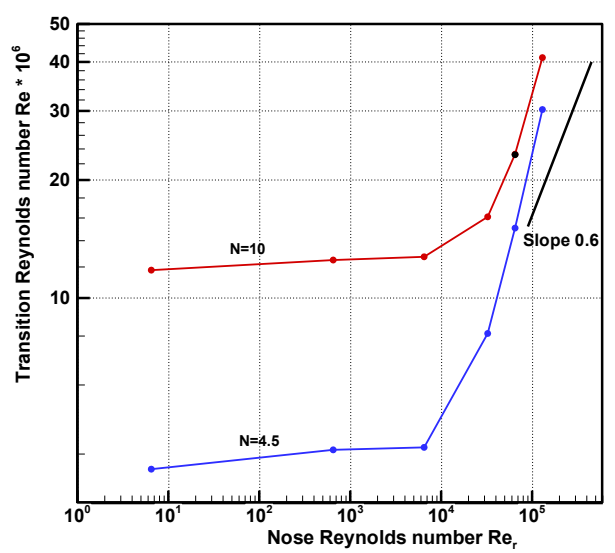

Figure 28. The transition Reynolds numbers obtained with $\mathrm{N}=10$ and 4.5 for different range of bluntness.



Figure 29. Eigenvalues from the linear stability computations for fast (blue) and slow (red) modes for the frequency $F=0.75 * 10^{-4}, r_{0}=0.05$ in.

American Institute of Aeronautics and Astronautics 
(a) $r_{0}=0.001$ in., $F=0.80^{*} 10^{-4}$

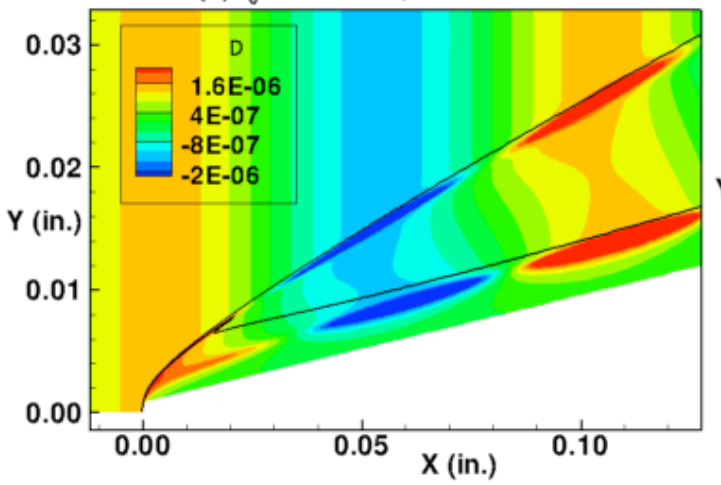

(b) $r_{0}=0.05$ in., $F=0.75^{*} 10^{-4}$

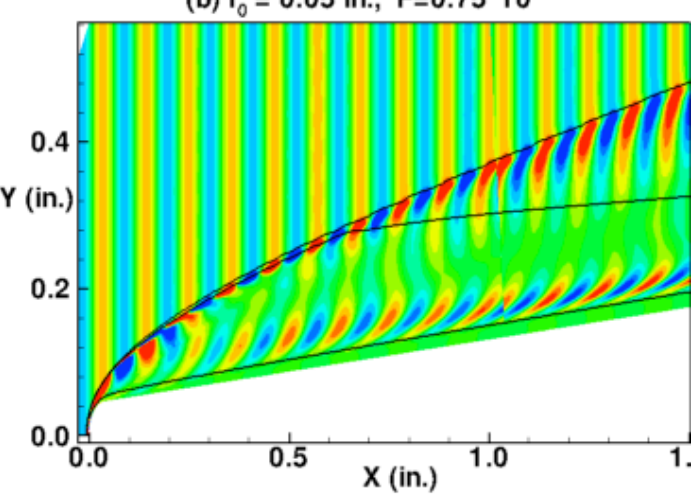

Figure 30. Contours of unsteady density fluctuations inside the boundary layer, near the nose region for two bluntness cases: (a) $r_{0}=0.001, F=0.80 \times 10^{-4}$ and (b) $r_{0}=0.05, F=0.75 \times 10^{-4}$.
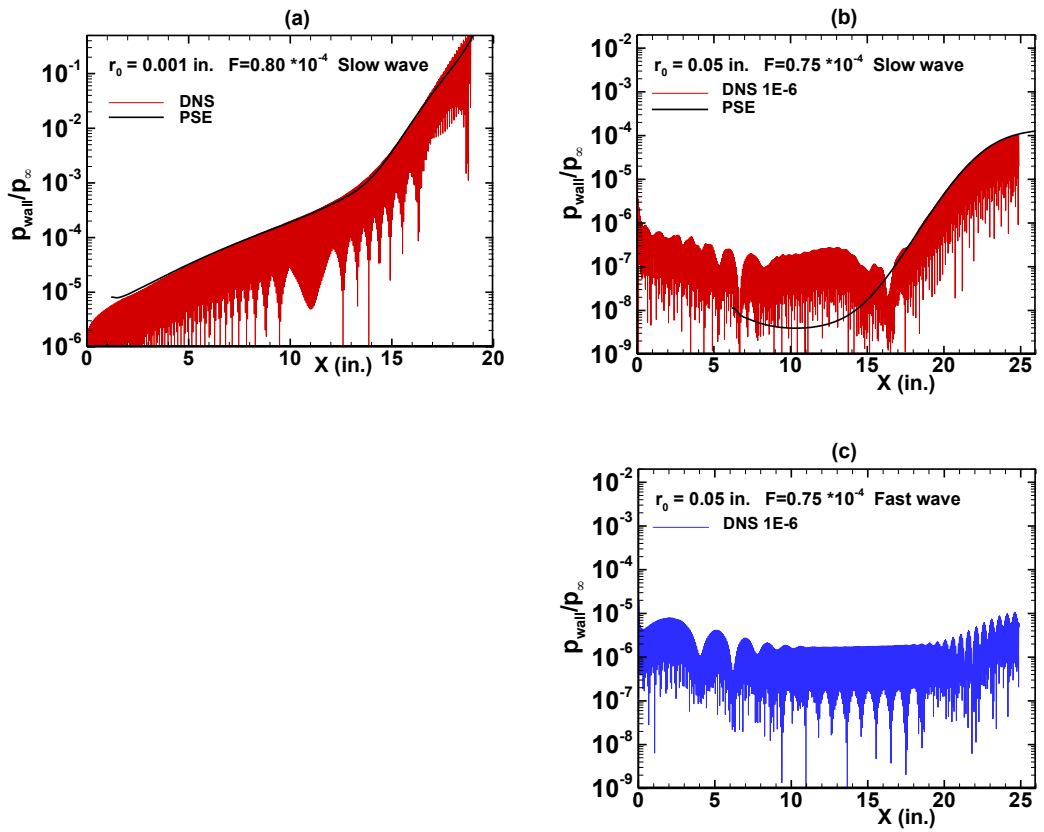

Figure 31. Amplitude of the pressure fluctuations on the wall and comparison with the PSE. (a) $\mathbf{r}_{0}=0.001$, slow wave, (b) $r_{0}=0.05$, slow wave and (c) $r_{0}=0.05$, fast wave.

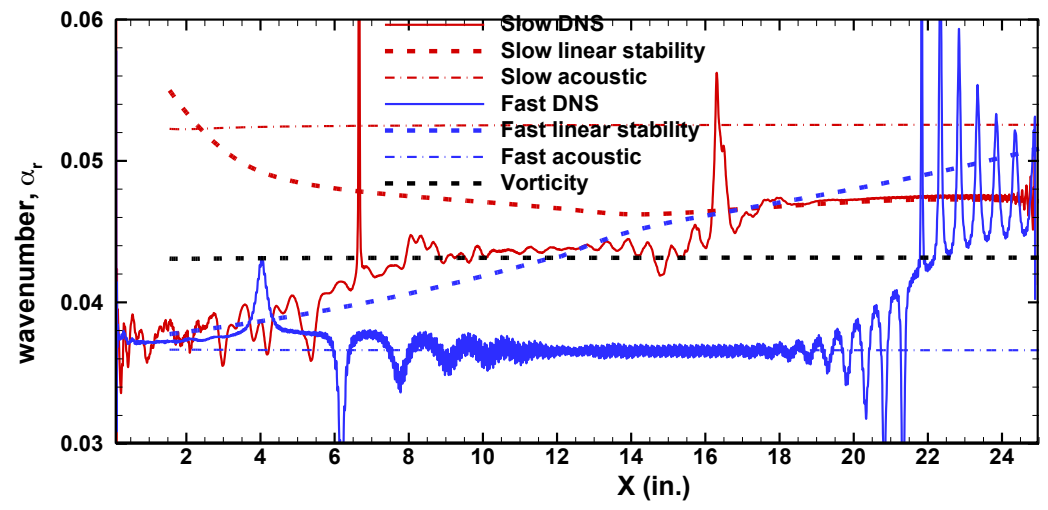

Figure 32. Wavenumber of the pressure fluctuations along the wall and comparison with the linear stability. $F=0.75 * 10^{-4}$. 

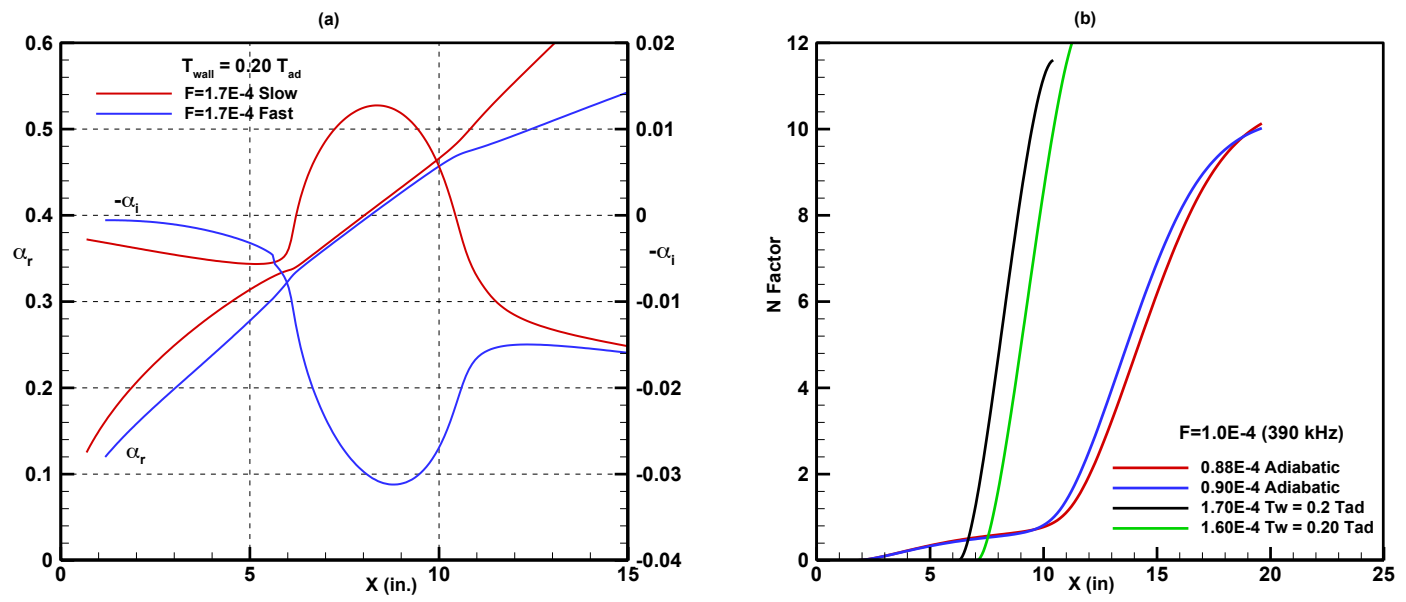

Figure 33. Eigenvalues $\left(\alpha_{r}, \alpha_{i}\right)$ for fast and slow modes for $F=1.7 * 10^{-4}$ and $(b) \mathrm{N}$-Factor curves.

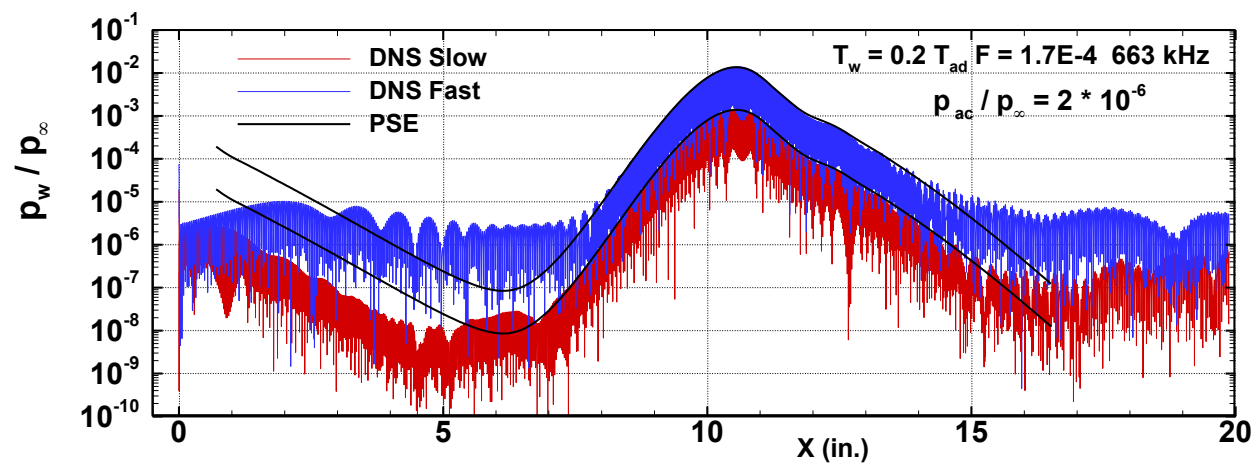

Figure 34. Amplitude of the pressure fluctuations on the wall generated by the slow and fast acoustic waves. $\mathbf{T}_{\mathrm{w}}=\mathbf{0 . 2} \mathbf{T}_{\mathrm{ad}}$.

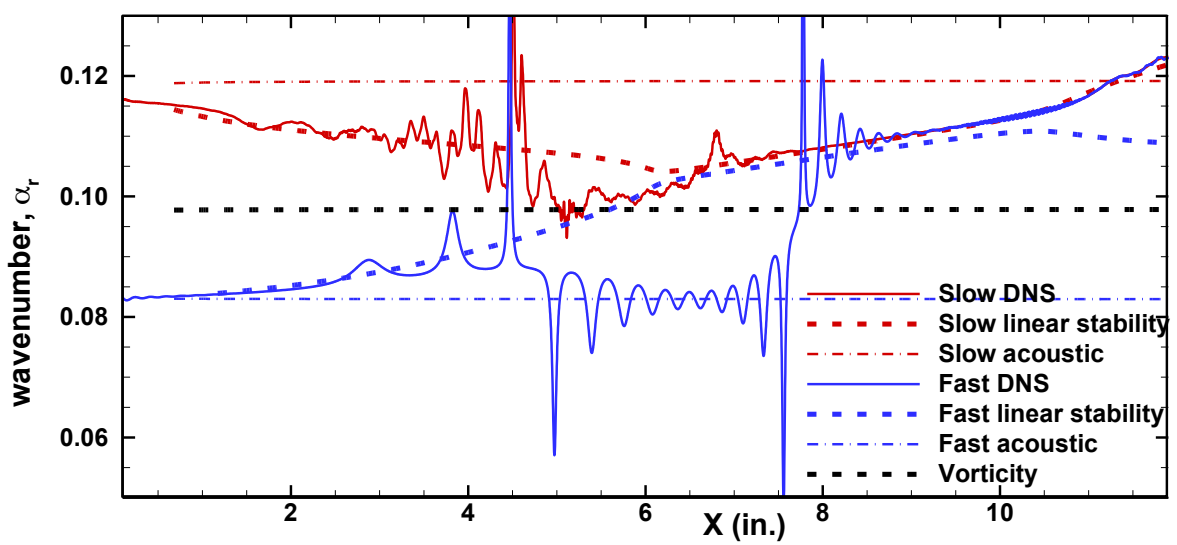

Figure 35. Wavenumber of the pressure fluctuations along the wall and comparison with the linear stability. $F=1.7 * 10^{-4}$. 


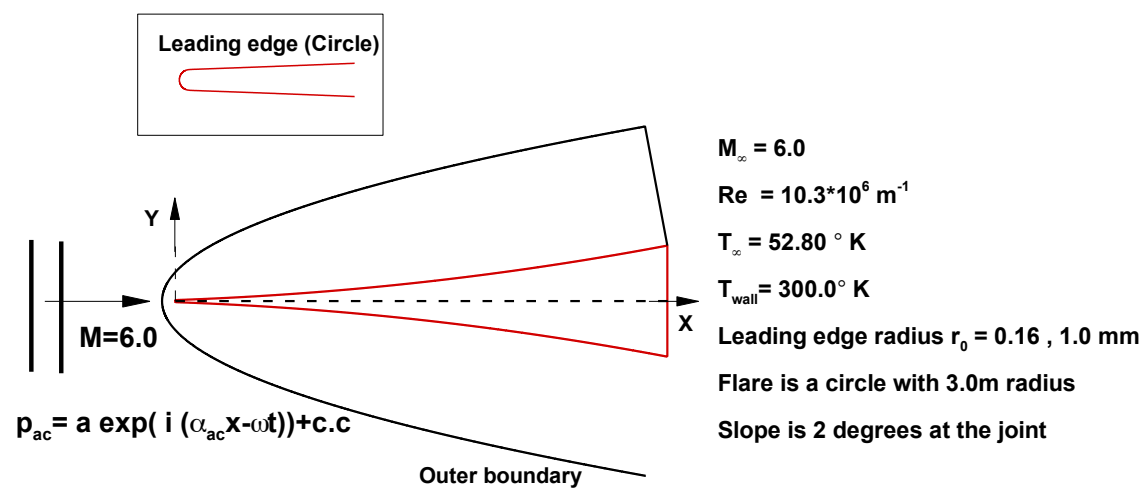

Figure 36. Schematic diagram of the computational model for flow over a circular flared cone.
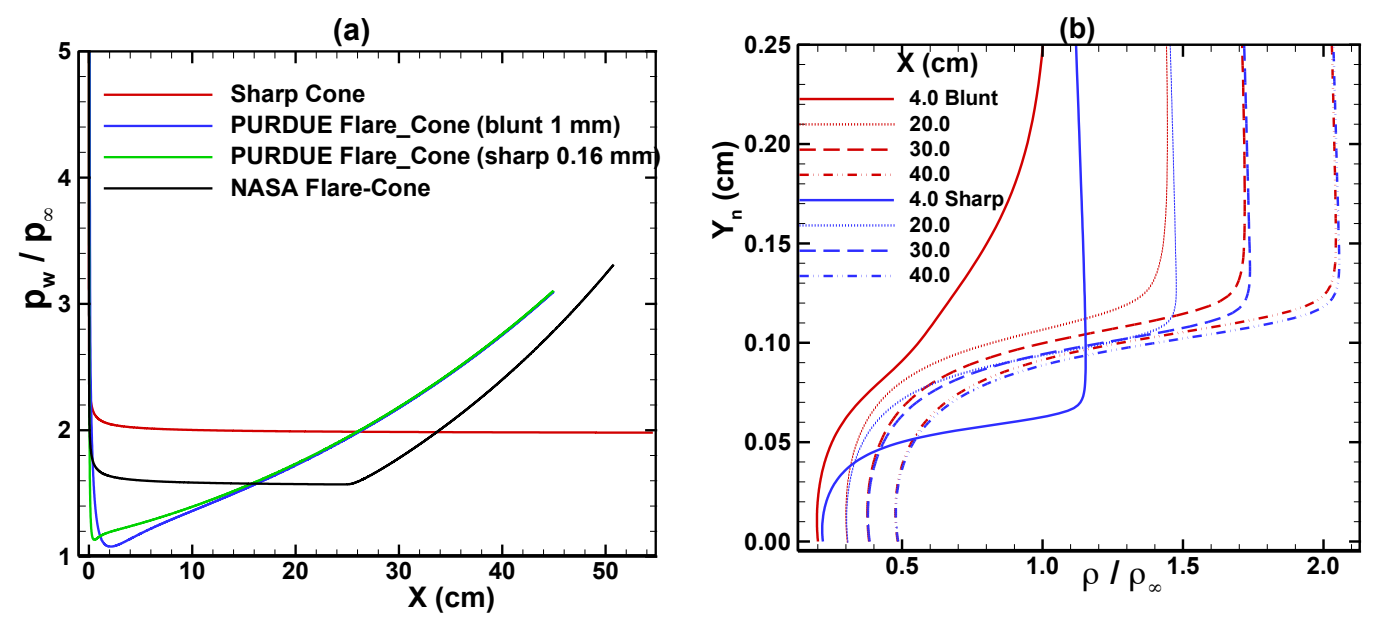

Figure 37. (a) Surface pressure distributions for straight and flare cone models and (b) boundary layer density profiles for the Purdue flared cones.

(a)

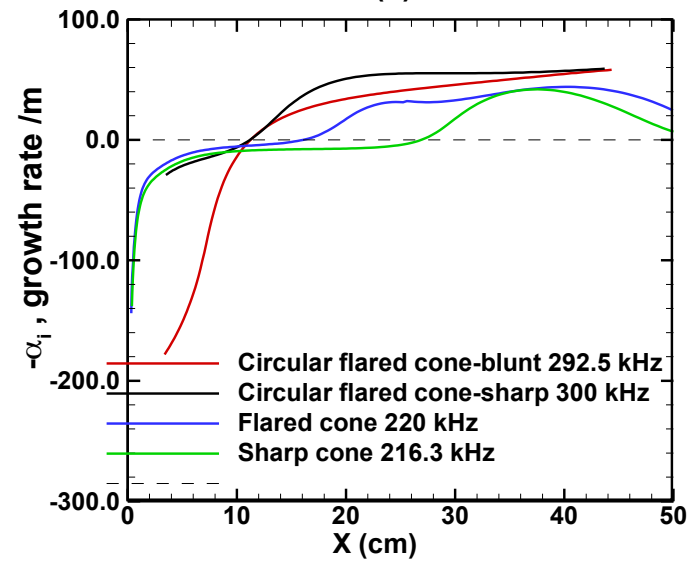

(b)

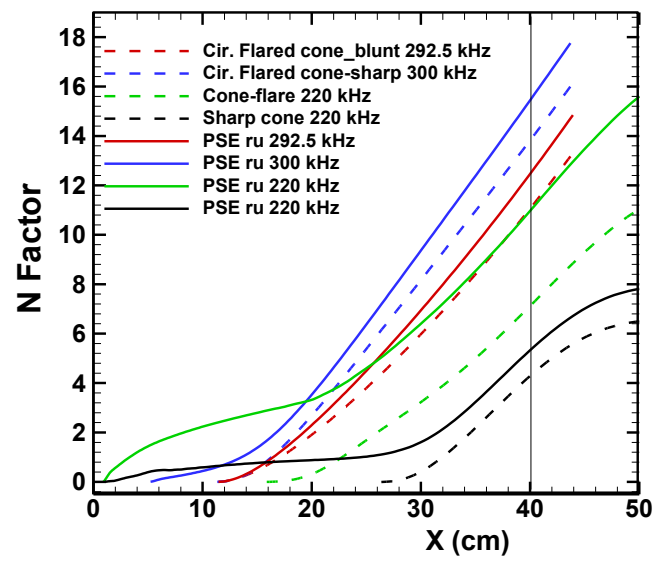

Figure 38. a) Comparison of the growth rates for the most amplified waves for different models and b) NFactors obtained from local and non-local PSE calculations. 

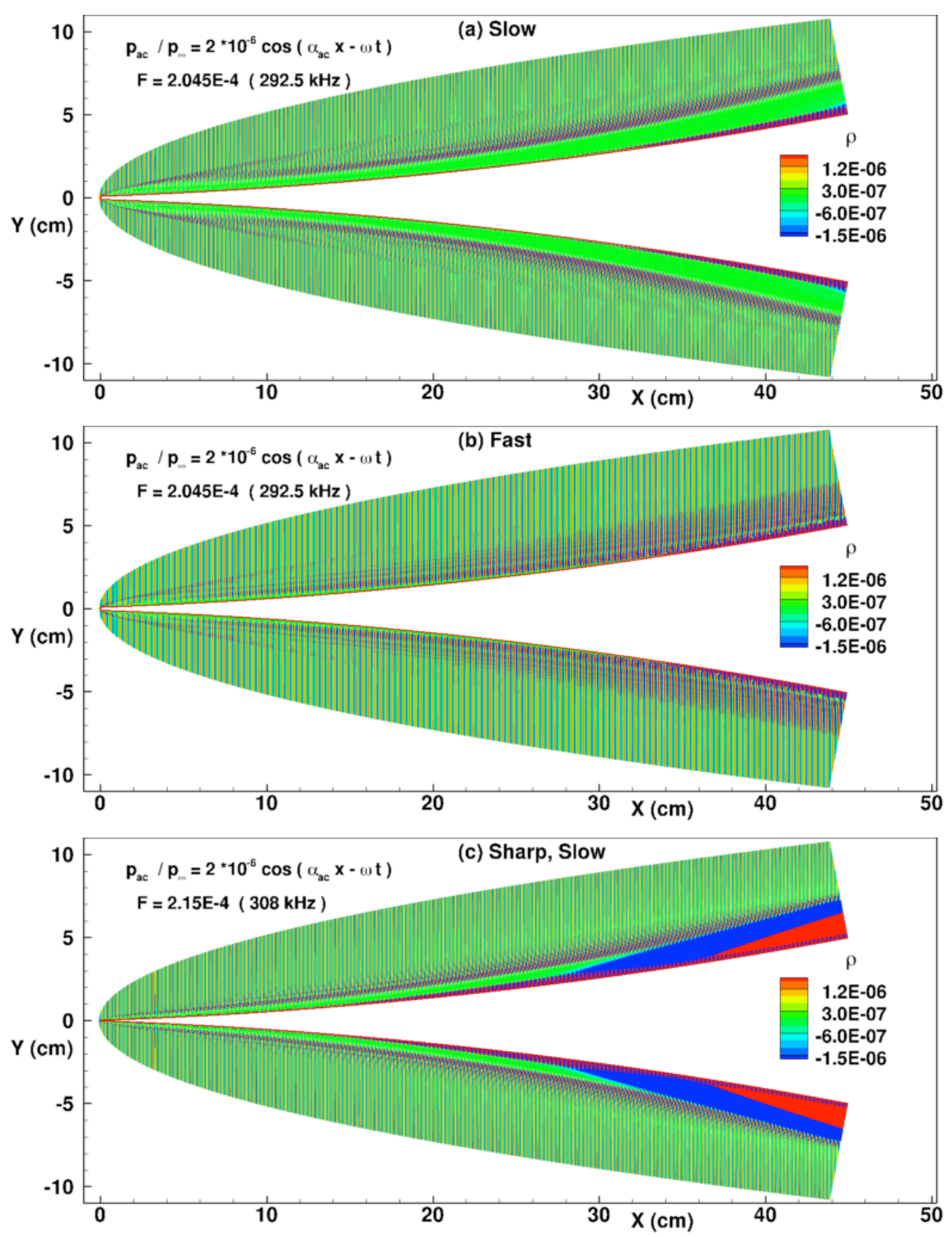

Figure 39. Density fluctuations generated by the interaction of a slow and a fast two-dimensional acoustic wave with the blunt and sharp circular flared cones. 
(a) Blunt, Slow

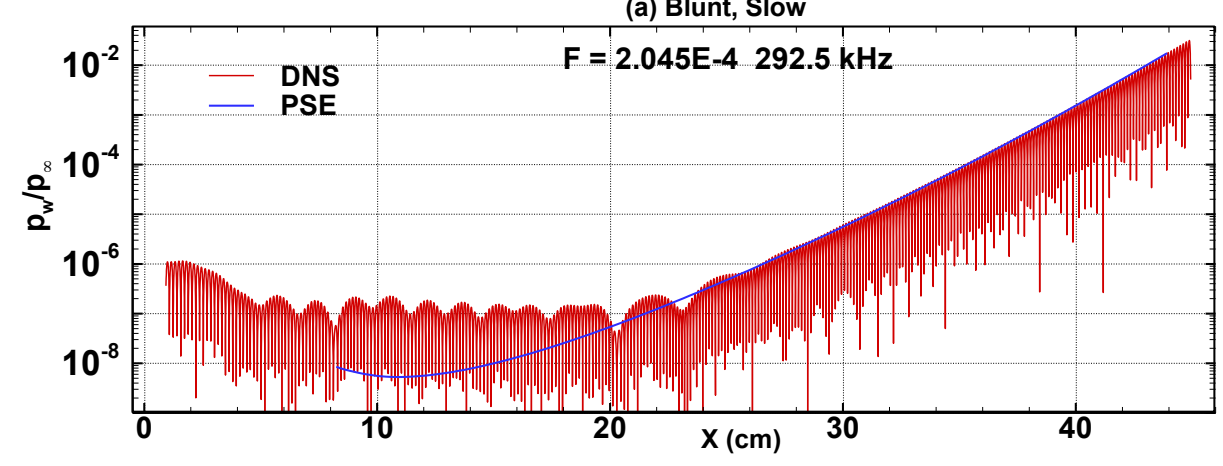

(b) Blunt, Fast
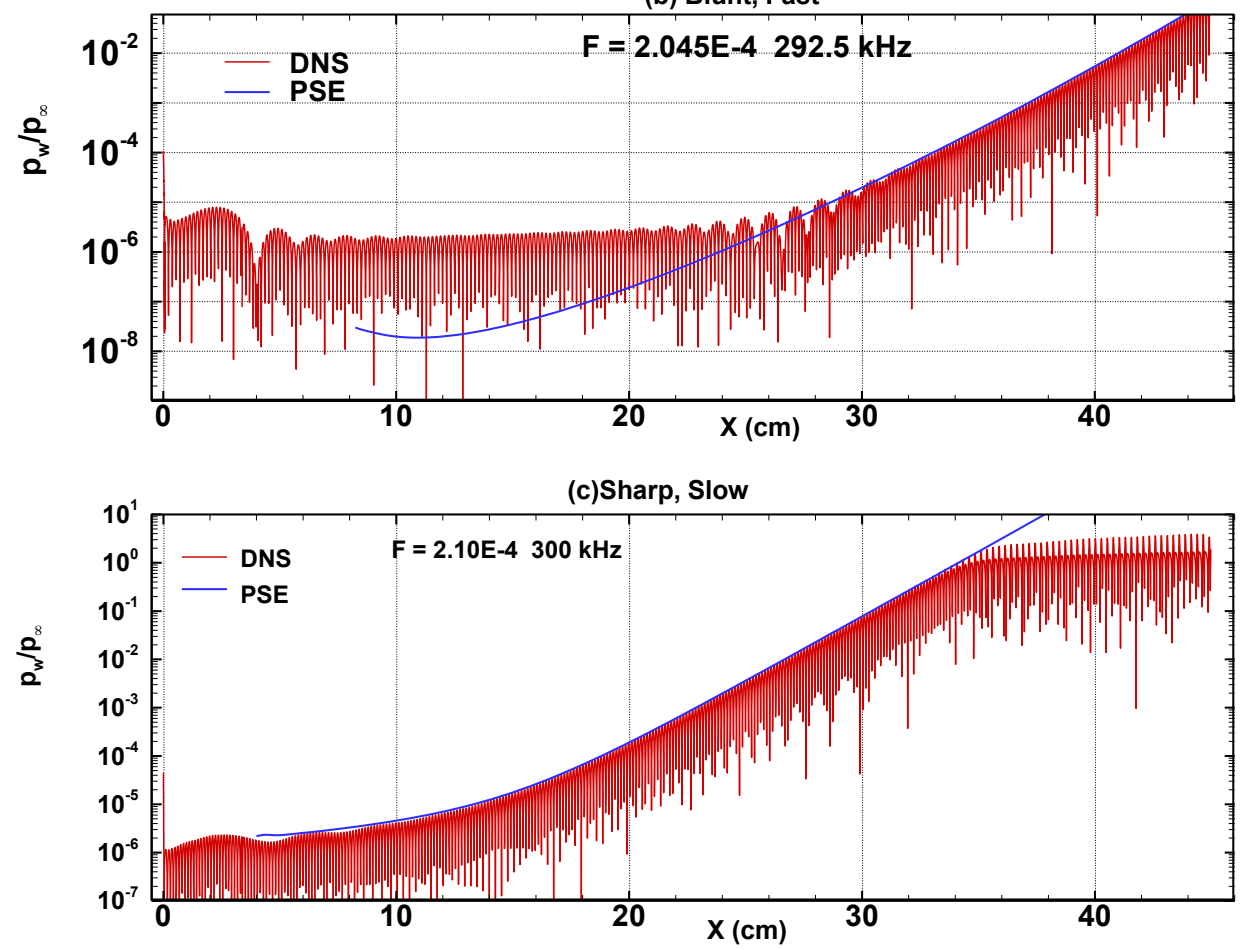

Figure 40. Wall-pressure fluctuations along the circular flared cone obtained from the DNS for a slow and fast acoustic wave. The results of the PSE calculations are shown for comparison. 

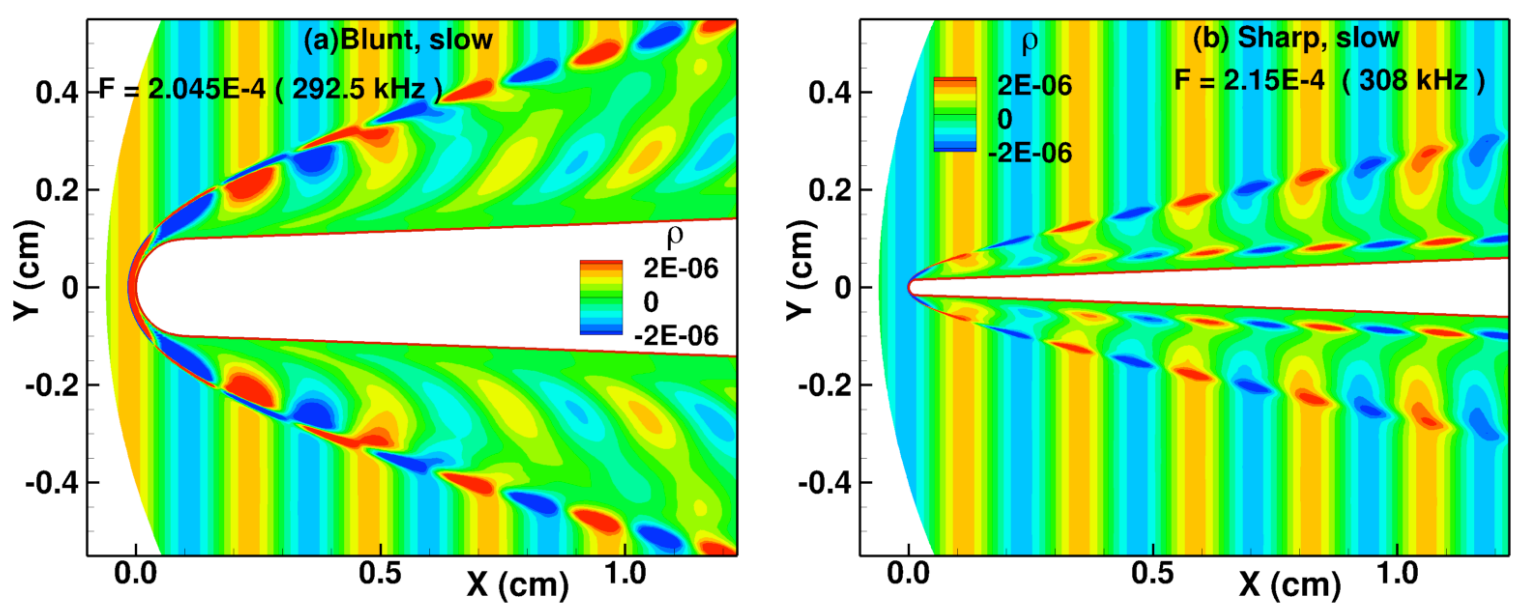

Figure 41. Density fluctuations generated by the interaction of a slow and a fast two-dimensional acoustic wave with (a) the blunt and (b) sharp circular flared cones.

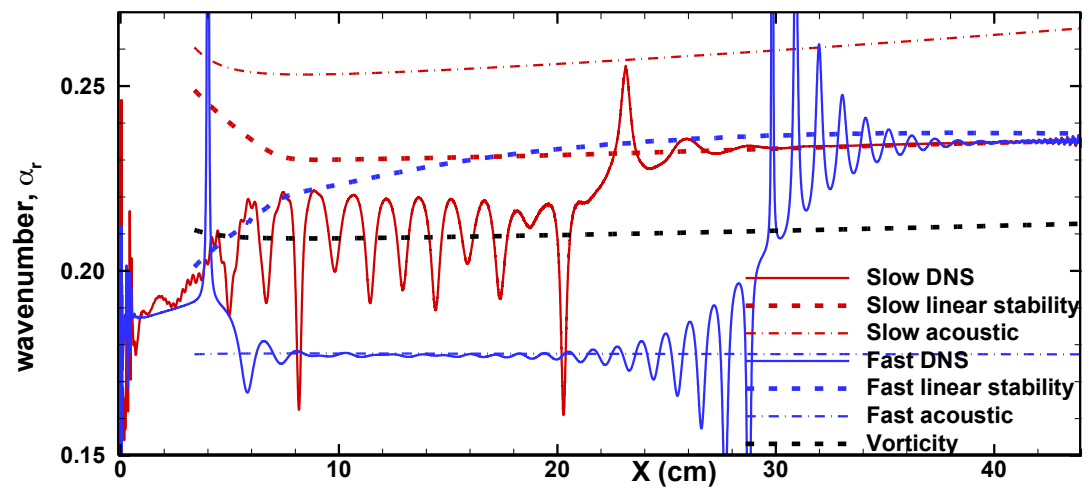

Figure 42. Wavenumber of the pressure fluctuations along the wall generated by two-dimensional acoustic waves and comparison with the linear stability for the blunt flared cone. $f=292.5 \mathrm{kHz}$.

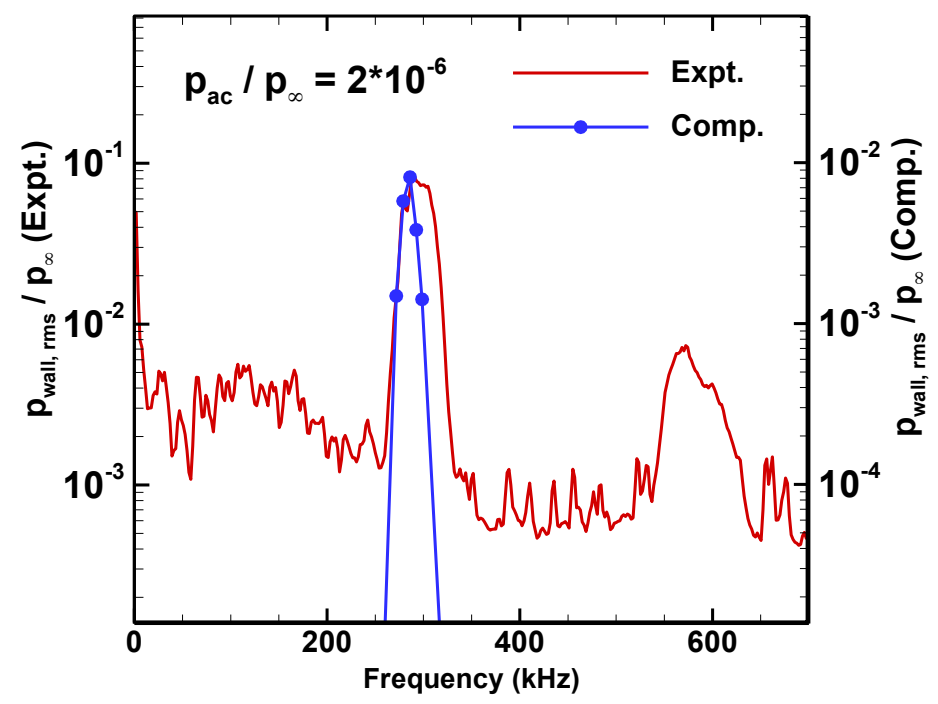

Figure 43. Comparison between the computed and measured wall-pressure spectrum for the circular flared cone at an axial location of $x=0.40 \mathrm{~m}$. Experimental data from Wheaton et. al. ${ }^{13}$ 


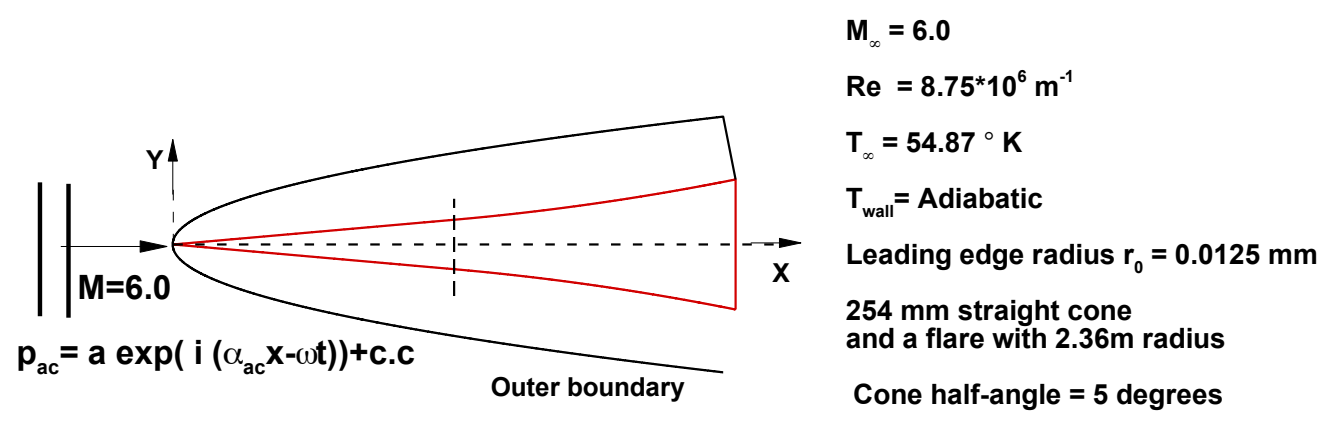

Figure 44. Schematic diagram of the computational model for flow over a sharp-tipped circular flared cone



Figure 45. Wall-pressure fluctuations along the sharp-tipped flared cone obtained from the DNS for a slow acoustic wave with frequency $f=220 \mathrm{kHz}$.



Figure 46. Comparison between the computed wall-pressure spectrum and a measured hot-wire voltage spectrum $^{10}$ for the sharp-tipped flared cone at an axial location of $x=40 \mathrm{~cm}$. Experimental data from Lachowicz et. al. ${ }^{10}$ 